\title{
Probing Phonons in Nonpolar Semiconducting Nanowires with Raman Spectroscopy
}

\author{
Kofi W. Adu, ${ }^{1,2}$ Martin D. Williams, ${ }^{3}$ Molly Reber, ${ }^{3}$ Ruwantha Jayasingha, ${ }^{3}$ \\ Humberto R. Gutierrez, ${ }^{4}$ and Gamini U. Sumanasekera ${ }^{3,5}$ \\ ${ }^{1}$ Physics Department, Altoona College, Pennsylvania State University, Altoona, PA 16601, USA \\ ${ }^{2}$ Materials Research Institute, Pennsylvania State University, University Park, PA 16802, USA \\ ${ }^{3}$ Department of Physics \& Astronomy, University of Louisville, Louisville, KY 40292, USA \\ ${ }^{4}$ Physics Department, Pennsylvania State University, University Park, PA 16802, USA \\ ${ }^{5}$ Conn Center for Renewable Energy Research, University of Louisville, Louisville, KY 40292, USA
}

Correspondence should be addressed to Kofi W. Adu, cxa269@psu.edu

Received 31 May 2011; Revised 27 July 2011; Accepted 13 August 2011

Academic Editor: Qihua Xiong

Copyright ( $) 2012$ Kofi W. Adu et al. This is an open access article distributed under the Creative Commons Attribution License, which permits unrestricted use, distribution, and reproduction in any medium, provided the original work is properly cited.

We present recent developments in Raman probe of confined optical and acoustic phonons in nonpolar semiconducting nanowires, with emphasis on Si and Ge. First, a review of the theoretical spatial correlation phenomenological model widely used to explain the downshift and asymmetric broadening to lower energies observed in the Raman profile is given. Second, we discuss the influence of local inhomogeneous laser heating and its interplay with phonon confinement on Si and Ge Raman line shape. Finally, acoustic phonon confinement, its effect on thermal conductivity, and factors that lead to phonon damping are discussed in light of their broad implications on nanodevice fabrication.

\section{Introduction}

Since the discovery of the Raman scattering process by Raman and Krishnan in 1928 [1] and the invention of the laser in 1960 by Maiman [2-4], Raman spectroscopy has morphed from standard macroprobe of bulk materials to single molecular detection [5-11]. The innovations in Raman spectroscopy have made it one of the most widely used techniques to probe nanostructures, beside transmission electron microscopy (TEM), scanning electron microscopy (SEM), Xray diffraction (XRD), and atomic force microscopy (AFM) as shown in Figure 1. The data in Figure 1 was collected from the ISI web of science site for the period of January 1996 to July 2010 on nanoscale characterization tools used in probing nanostructures [12]. Even though probing individual nanostructures by Raman spectroscopy at nanoscale is limited by its low spatial resolution (diffraction limit of the objectives), recent developments in nano-Raman spectroscopy, including scanning near-field optical microscopy (NSOM), tip enhanced Raman spectroscopy (TERS), and surface enhanced Raman spectroscopy (SERS), have made single-molecule detection possible [5-11, 13-17], especially using SERS and TERS. While NSOM is based on the principle of optical tunneling of evanescent waves, TERS and SERS are based on excitation of surface plasmons on the surface of nanometals. The process arises when free electrons on the surface of a metal oscillate collectively in resonance with the oscillating electric field of the incident light wave. The interaction between the surface charge and the electromagnetic field helps concentrate the light on the surface of the metal, leading to electric field enhancement. Irrespective of such great innovative techniques in nano-Raman spectroscopy, nearly $84 \%$ (see Figure 1) of Raman characterization use conventional Raman spectroscopy to probe nanoscale samples [12]; that is, probing ensembles of nanostructures instead of individual nanostructures.

The dramatic surge in the use of Raman spectroscopy in probing nanostructures is mainly due to the immense information (such as amorphization, defects, sample temperature (local heating), size distribution, strain effects, phonon confinement, type of doping, phase separation, carrier concentration, and mobility) [24-38] that can be readily acquired 




FIgURE 1: Number of records of the listed nanocharacterization tools used in probing nanostructures in the period of January 1996 to July 2010 (Source: ISI Web of Science, http://apps.isiknowledge $. \mathrm{com} /)[12]$.

from analysis of the peak-frequency and line width of the Raman profile. Additionally, (i) the nondestructive nature of the Raman probing technique, (ii) limited or no sample preparation, (iii) simultaneous in situ measurement, (iv) submicron spatial resolution when coupled with AFM (TERS) or SEM, and (v) very high sensitivity when coupled with surface enhanced/resonance spectroscopy have made Raman spectroscopy an easy-to-use instrument for quick sample screening. Thus, Raman spectroscopy has been used extensively to probe optical phonons, surface phonons, acoustic phonons, and antenna effects in nanowires.

In this paper, we concentrate on recent developments in optical and acoustic phonon confinement in nonpolar semiconducting nanowires, using $\mathrm{Si}$ and $\mathrm{Ge}$; as examples, since many of the concepts that we discuss here are applicable to any related systems. Other phenomena in nanowires such as antenna effects and surface phonons are not covered here. In this chapter, we will review optical and acoustic phonon properties using $\mathrm{Si}$ and $\mathrm{Ge}$ nanowires as prototype and the new phonon dispersion due to radial (circumferential) confinement. Additionally, we will review physical properties such as thermal conductivity that are significantly influenced by phonon confinement.

Our understanding of the bulk properties of crystalline and amorphous Si and Ge continue to be of immense importance to the development of electronic, optoelectronic, photovoltaic, and other devices, as well as the society at large. The past two decades have seen scientific advances in low-dimensional systems by reducing the three-dimensional bulk system (3D) to two-dimensional thin-film systems (2D) to one-dimensional and quasi-one-dimensional (1D) systems of nanowires and nanotubes [39-64] and to zero-dimensional (0D) systems of quantum dots [39-41].

Following the growth of nanowires using pulsed laser vaporization in 1998, different synthesis techniques have been developed using the vapor-liquid-solid mechanism (VLS) [65-79]. These have led to intense research activities due to the unique properties of the nanowires compared to their bulk counterparts, and several applications of nanowires including sensors, transistor and logic gates, electrooptics, thermoelectric, photovoltaic, electrochemical capacitors, and batteries have been proposed. Most of these applications take advantage of quantum confinement effects at small diameters, which for $\mathrm{Si}$ and Ge occur at diameters $\leq 20 \mathrm{~nm}[44-47,80]$. Both theoretical [81-84] and experimental $[83,85-88]$ evidence of the effects of confinement on the electronic states of nanowires have been reported for $\mathrm{Si}$ and other nanowires [89-91]. These reports indicate a transition from indirect band gap to direct band gap in the nanowires where the band gap scales with diameter as $\sim D^{-1.7}[83,84,88,92,93]$. Most studies of nanowires have focused on the electronic states; however, the importance of phonon states in nanowires cannot be overemphasized. Most of the electronic properties are intricately influenced by phonon scattering since it is the dominant scattering mechanism at high temperatures. The influence of phonon confinement, geometry, and surface roughness of nanowires on thermal conductivity, specific heat capacity, and electron mobility has been demonstrated theoretically and experimentally [94-101]. Different confinement models including lattice dynamic simulation [102] and spatial correlation (phenomenological) [12, 44-47, 80, 103-111] model have been used to calculate phonon dispersion and Raman line shape profiles of nanowires, respectively. We will discuss the phenomenological model later. However, readers who are interested in the lattice dynamic calculation should see [102].

Majority of the optical phonon confinement reports in the literature for semiconducting nanowires of (a) Si, Ge, (b) III-VI, II-VII compounds, and (c) oxide compounds have analyzed their results using the spatial correlation model developed by Richter and coworkers [106] and further extended by Campbell and Fauchet [107]. Application of this model to experimental data and the influence of laser flux on the Raman line shape will be discussed here.

Small diameter semiconducting nanowires exhibit phonon confinement due to the long length of the phonon wavevector $q$ compared to the radius of the nanowire, which violates the $q=0$ selection rule, permitting multiple wavevector of various $q<1$ to contribute to the phonon linshape. Based on the spatial correlation model first proposed by Richter et al. [106] and expanded further by Campbell and Fauchet [107], it has been argued that restriction of the phonon wave function within a particle of size $D$ results in an uncertainty $\Delta q \sim \pi / D$ in the phonon wavevector of the zone-center optical phonon and a corresponding uncertainty $\delta \omega$ in its wavenumber [112]. Consequently light scattering takes place from quasi-zone-center optical phonons with wavevector $q$ up to $\pi / D$. This is in fact valid only for weak phonon localization and the effect diminishes as $q$ approaches $\pi / D[110]$.

Germanium and silicon belong to the cubic $\mathrm{O}_{\mathrm{h}}{ }^{7}$ space group and crystallize into the $\left(\mathrm{F} 4_{1} / \mathrm{d} 32 / \mathrm{m}\right)$ diamond lattice. The conventional lattice of diamond consists of two interpenetrating face-centered cubic ( $\mathrm{fcc}$ ) atomic lattices which 


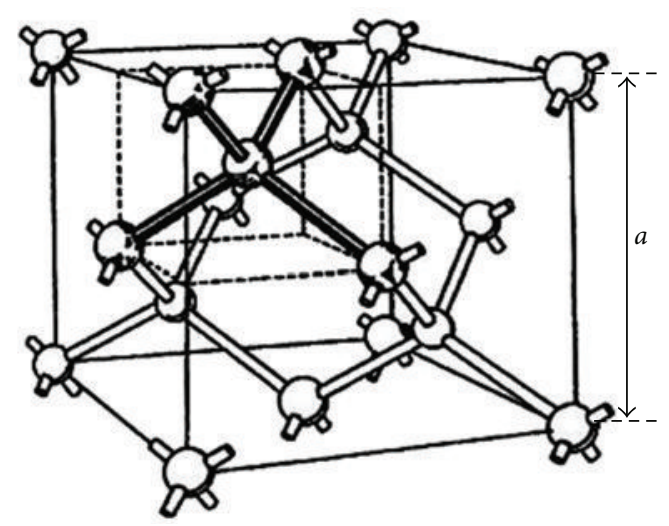

(a)



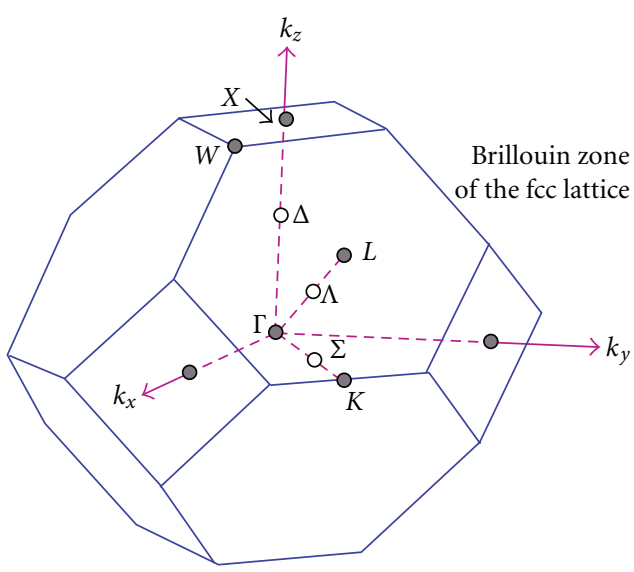

(b)

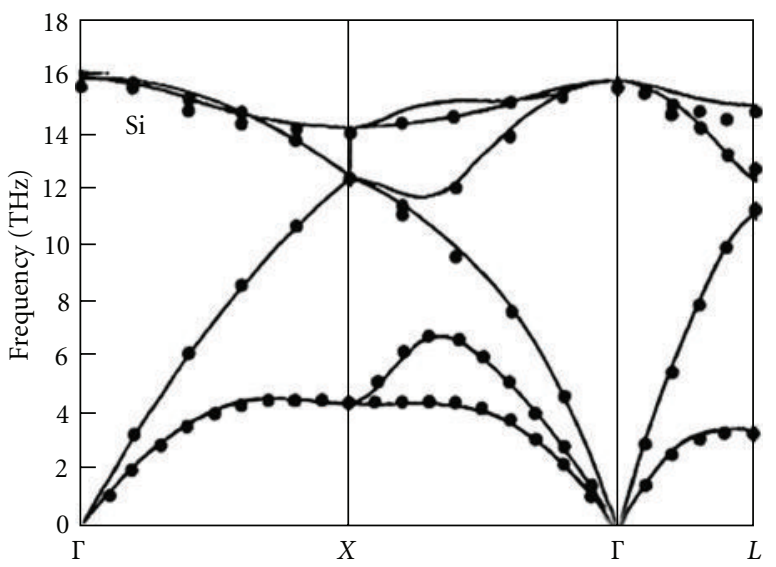

(c)

FIgURE 2: (a) Conventional cubic unit cell for the diamond crystal structure ( $\left.\mathrm{F} 4_{1} / \mathrm{d} 32 / \mathrm{m}\right)$. Each atom is tetrahedrally bonded to four nearest neighbors. The lattice parameter of the conventional unit cell containing four atoms, $a=0.5431 \AA$ ( $\mathrm{Si}$ ) and $a=0.5658 \AA$ (Ge), (b) Brillouin zone for the face-centered space lattice showing the positions of several high-symmetry axes and points [18, 19]. (c) Phonon dispersion curves of Ge [20] (left) and Si [20] (right) along high-symmetry directions. The dots are neutron scattering data [19, 21, 22], and the solid lines are calculations using up to the sixth nearest neighbor interactions in a Born-von Kármán force constant model [20]. The primitive cell contains two atoms; therefore, three optical branches (2TO and 1LO) and three acoustic branches (2TO and 1LO) are observed. These branches are degenerate at the $\Gamma$ point.

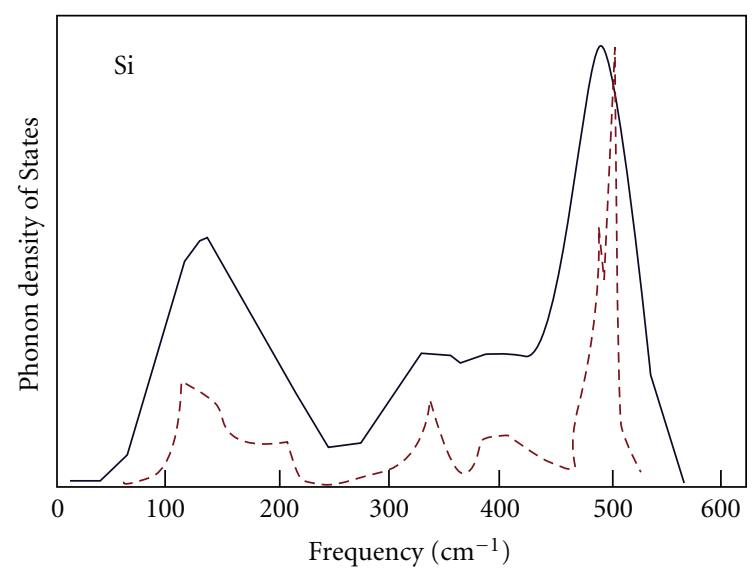

(a)

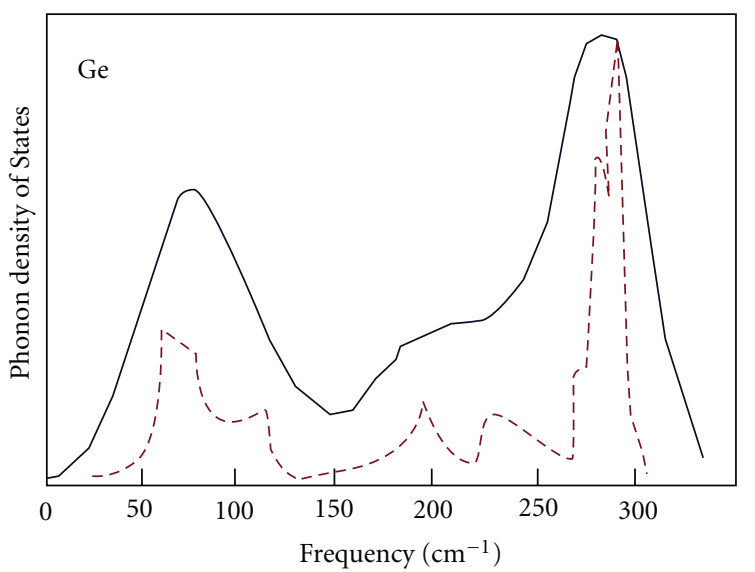

(b)

FIgURE 3: Calculated phonon density of state for Si (a) and Ge (b). Solid lines represent a highly disordered material, and the dashed lines represent the crystalline material [23]. 


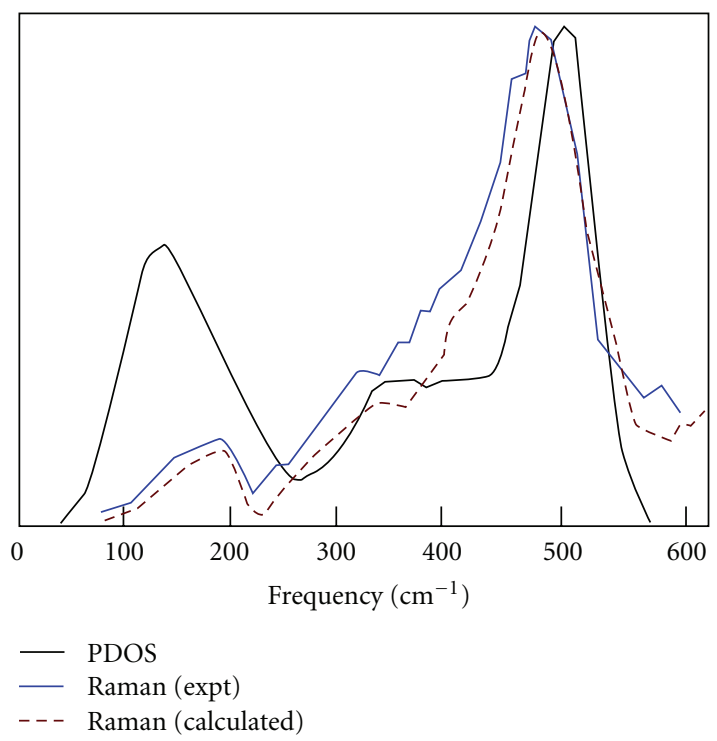

Figure 4: Comparison of experimental Raman profile (thin solid line) and calculated first-order Raman band of highly disordered Si (dashed line). The bold solid line is the calculated phonon density of states (PDOS) for highly disordered Si. The experiment and calculated Raman spectra are in good agreement. The difference in the PDOS and the calculated Raman band shows the importance of matrix element effects [23].

are displaced a quarter of the body diagonal of the conventional cubic cell with respect to each other. Each atom is tetrahedrally bonded to four nearest neighbors. The 3-dimensional unit cell of a tetrahedrally bonded diamond lattice is shown in Figure 2(a), and the corresponding Brillouin zone indicating the positions of several high-symmetry axes $(\mathbf{X}, \mathbf{L}, \mathbf{W}, \mathbf{K})$ and points $(\Gamma, \Lambda, \Delta, \Sigma)$ is shown in Figure $2(\mathrm{~b})$ $[18,117]$. The phonon dispersion curves along some of these high-symmetry axes for crystalline $\mathrm{Si}$ and Ge, obtained from neutron scattering data $[19,21]$ (dots) and a fit to the data using (up to the sixth nearest neighbor) the Bornvon Kármán force constant model (solid line), are shown in Figure 2(c) [19-22] right and left, respectively. At the points in the Brillouin zone where the phonon dispersion curves flatten, $d \omega / d k \sim 0$, and peaks appear in the phonon density of states. However, in highly disordered systems, these features in the phonon density of states are observed to be broadened. Shown in Figure 3 are the calculated phonon density of states for bulk Si (a) and Ge (b). The dashed and solid lines in the figure represent crystalline and highly disordered systems [23], respectively. Note that the disorder introduces both smearing of the sharp peaks in the phonon density of states and disappearance of the fine structures associated with the crystalline state; however, the three main broad features remain. Interestingly, strong disorder does not destroy the main features of the phonon density of states associated with the crystalline system.

If we compare the first order Raman spectra of disordered and crystalline $\mathrm{Si}$, we observe a sharp Lorentzian peak at $\sim 520 \mathrm{~cm}^{-1}$ of full width at half maximum (FWHM) of $\sim 4 \mathrm{~cm}^{-1}$ for the crystalline state. Strongly disordered Si on the other hand exhibits a Raman spectrum that approaches the calculated phonon density of states shown in Figure 3(a). In this case, the peak at $\sim 520 \mathrm{~cm}^{-1}$ now exhibits a FWHM of $\sim 100 \mathrm{~cm}^{-1}$, that is, $\sim 25$ times broader than the crystalline bulk. In Figure 4, the calculated phonon density of states (thick solid line), Raman spectrum (thin solid line) and calculated Raman spectrum (dashed line) of a highly disordered Si are compared [23]. It is clear that the experimental and the calculated Raman data are in reasonably good agreement. It can also be inferred that the matrix element effects are important if a truly quantitative analysis of the data is desired. Nevertheless, the Raman spectrum of highly disordered system can provide very important phonon density of states information throughout the entire frequency range.

The first Brillouin zone is the smallest volume entirely enclosed by planes that are the perpendicular bisectors of the reciprocal lattice vectors drawn from the origin. The region in $k$-space that low- $k$ phonons can occupy without being diffracted is called the first Brillouin zone. It contains phonons with $k$ values from 0 to $\pi / a$, where $a$ is the lattice parameter. For monoatomic solids with two atoms per primitive cell such as diamond, magnesium, $\mathrm{Si}, \mathrm{Ge}$, or diatomic compounds such as GaAs, there are three optic phonon branches in addition to the three acoustic phonon branches. In compounds with a greater number of atoms and complex crystal structures, the number of optical phonons is more than three. If the crystal unit cell contains $N$ atoms, then $3 N$ degrees of freedom result in 3 acoustic phonons and $3 N-3$ optical phonons.

Phonons can propagate in the lattice of a crystal as a wave and their dispersion depends on their wavelength [118]. For a given nanowire material, the confinement effects may exhibit different asymmetric broadening and shifts, depending on the symmetries of the phonon and their dispersion curves. There is also dependence on the excitation energy such that phonons of certain symmetry are more responsive to resonance conditions [119]. Transverse phonon confinement in nanowire is due to their one-dimensional geometry. The wavevector is unrestricted in the longitudinal $(z)$ direction, but it is confined and quantized in the tangential (radial) direction as illustrated in Figure 5.

In bulk crystalline materials, Raman spectroscopy and other optical techniques can only probe zone center phonons. Absence of lattice periodicity in nanocrystalline materials relieves this $q=0$ selection rule. In general, for semiconducting nanowires, phonon confinement is not the only effect linked to diameter. Electronic states of semiconducting-nanowires can be tuned via the wire diameter $D$, as the band gap should scale approximately as $1 / D[120]$.

The organization of this chapter is as follows. We will discuss in Section 2 the spatial correlation phenomenological model for optical phonons and relate it to experimental data from Raman spectroscopy of confined optical phonons in $\mathrm{Si}$ and Ge nanowire in Section 3. In Section 4, we will discuss the influence of local inhomogeneous laser heating on the Raman profile of Si and Ge nanowire. Acoustic phonon confinement and its effect on thermal conductivity will be presented in Section 6 and Section 7 will be devoted to summary of the main points presented in this paper and suggestions for future research in phonon confinement. 




(a)

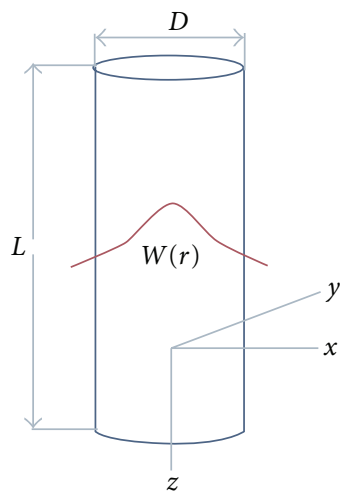

(b)

Figure 5: (a) Optical phonon dispersion $\omega(q)$ of Si with the first order Raman scattering phonon frequencies extend throughout $\Delta \omega$. Raman active wavevectors of a nanowire with diameter $D$ lie within $\Delta q$. (b) Schematic of nanowire of diameter $(D=2 r)$ and length $L$ and the confined phonon wave function, $W(r)$.

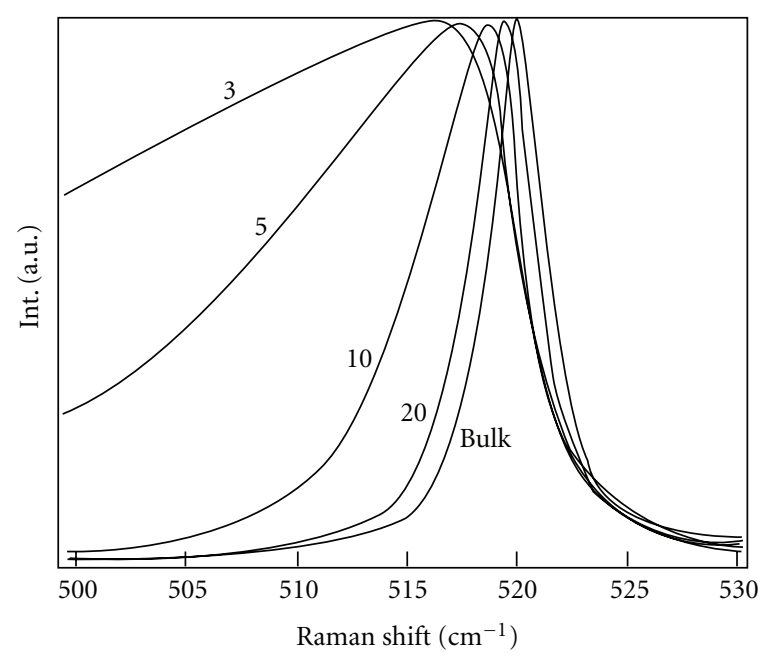

(a)



(b)

Figure 6: (a) A plot showing the finite-size-induced phonon confinement effect (downshift and asymmetric broadening to lower energy) for different diameters of Si nanowires and the Lorentzian line shape of the bulk, for comparison. (b) Comparison of the Raman line shape of $8 \mathrm{~nm}$ diameter Si nanowires, taking diameter distribution into account and without diameter distribution. The peak downshift $\sim 1 \mathrm{~cm}^{-1}$ and the line width broadened $\sim 30 \%$.

\section{Theoretical Models of Phonon Confinement in Nanowires}

2.1. Spatial Correlation Model of Raman Scattering for Confined Optical Phonons in Nanowires. Richter and coworkers [106] were the first to propose a phenomenological phonon confinement model (sometimes called Richter model) to explain the experimental observation of a downshift and asymmetric broadening of the first-order Raman profile of crystalline nanostructures. Their approach naturally leads to relaxation of the conservation of the bulk crystal momentum in the scattering process. Assuming a spherical nanocrystalline shape and using the diameter as an adjustable parameter, they fitted the Raman line shape of a film of small Si nanocrystals. Their model was further extended by Campbell and Fauchet [107] who considered other nanocrystalline shapes (i.e., wires and platelets).

For Si and Ge, the longitudinal optic (LO) and the tangential optic ( $\mathrm{TO}$ ) modes are degenerate at the $\Gamma$ point (zone center). Conservation of the phonon momentum in crystalline $\mathrm{Si}$ and Ge produces a Raman active mode of the optical phonons from only the zone center $(q=0)$ at $520 \mathrm{~cm}^{-1}$ and $300 \mathrm{~cm}^{-1}$ for $\mathrm{Si}$ and $\mathrm{Ge}$, respectively. In nanowires, the transverse phonons are confined in the two orthogonal directions perpendicular to the nanowire axis. This allows a greater range of phonon modes to contribute to the Raman 


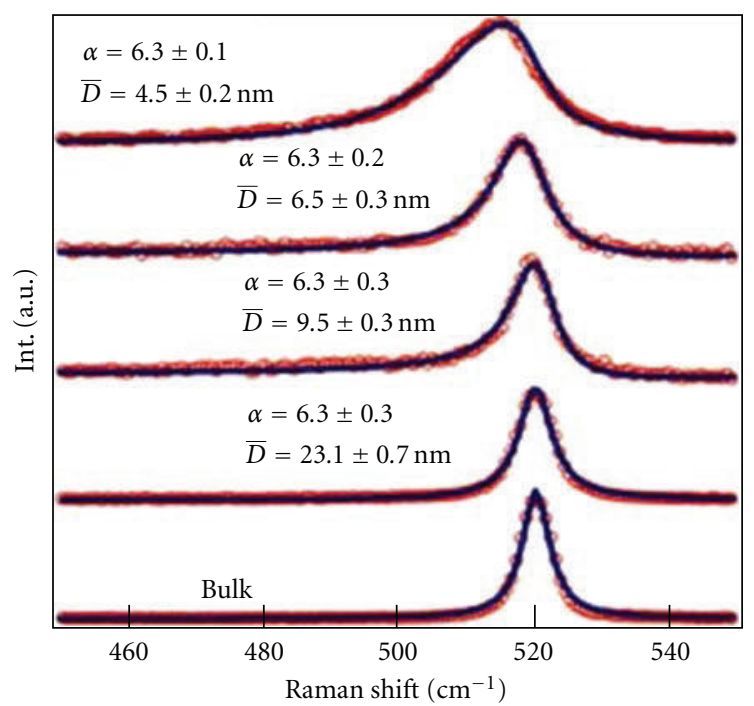

(a)

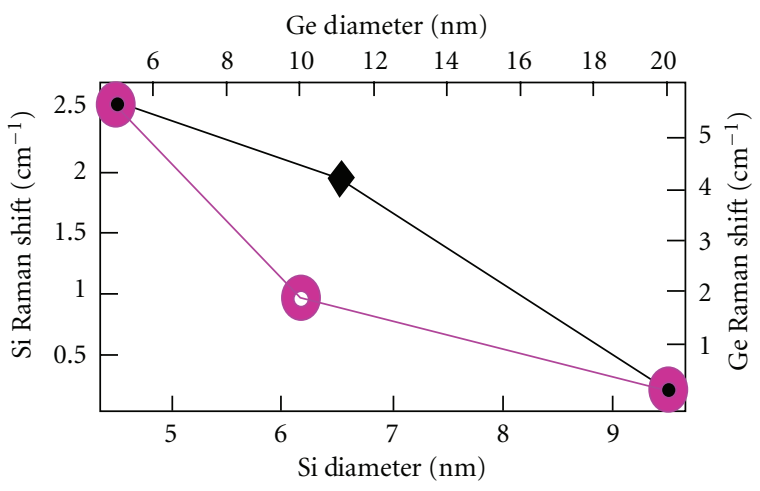

(b)



(c)

FIgURE 7: (a) Raman spectra showing the first-order Raman band peaks in four ensembles of Si nanowires. The bulk Si (001) spectrum is also shown. Experimental data: red open circles, least-square fit: black solid lines. $\alpha$ is the attenuation factor of the phonon (the width of the Gaussian phonon quantum confinement function) at the real boundary $r=D / 2, \bar{D}$ is the most probable diameter. (b) Comparison of experimental Raman shift of TO phonon in Si [47] and Ge nanowires [80] for different diameters. Top: absolute shift Si (diamond), Ge (circles); bottom: nanowire Raman shift relative to bulk. Note that the Ge diameter range is twice that of Si.

scattering process. In fact, bulk phonons of wavevector up to $q \sim 1 / D(D$ is diameter of the nanowire $)$ contribute to the first-order Raman spectrum.

The Richter model line shape function of the first-order Raman profile of a nanowire expressed in terms of the transverse phonon wavevector $\left(q_{\perp}\right)$ can be written as [44-47, 80, $104,106,107,113,121-123]$

$$
I_{N W}(\omega)=A_{o} \int_{o}^{q_{\max }} \frac{\left|C\left(0, q_{\perp}\right)\right|^{2}}{\left[\omega-\omega_{o}\left(q_{\perp}\right)\right]^{2}+(\Gamma / 2)^{2}} 2 \pi q_{\perp} d q_{\perp}
$$

where $A_{o}$ is an adjustable parameter, $\omega$ is phonon frequency measured relative to the laser line, $\omega_{o}\left(q_{\perp}\right)$ is the bulk transverse phonon frequency, $\Gamma$ is the FWHM of the bulk phonon Raman profile, and $\left|C\left(0, q_{\perp}\right)\right|^{2}$ is the spectral weighting function expressed as

$$
\left|C\left(0, q_{\perp}\right)\right|^{2} \sim \exp \left[-\frac{1}{2}\left(\frac{q_{\perp} D}{\alpha}\right)^{2}\right]
$$

$D$ is the diameter of the nanowire and $\alpha$ is a dimensionless adjustable parameter. Figure $6(\mathrm{a})$ shows the evolution of the Raman profile with diameter $(20 \geq D \geq 3)$ of the $520 \mathrm{~cm}^{-1}$ optical phonon of Si nanowire, calculated using (1). Here, $\Gamma=4.5 \mathrm{~cm}^{-1}$ and $\alpha=6.3$ were used in the calculations [53]. Since most Raman scattering experiments are performed on ensembles of nanowires, any analysis using the Richter model should take into account the diameter distribution $F(D)$ of the ensemble nanowires. This modifies the Richter model by introducing a second integral due to the diameter distribution as

$$
I_{N D W}(\omega, \bar{D})=\int_{o}^{\infty} F(D) I_{N W}(\omega, D) d D,
$$

where $\bar{D}$ is the ensemble most probable diameter and the rest of the parameters are as defined previously. Figure 6(b) shows a comparison of calculated Raman line shape of $8 \mathrm{~nm}$ Si nanowire with (solid line) and without (dash line) 


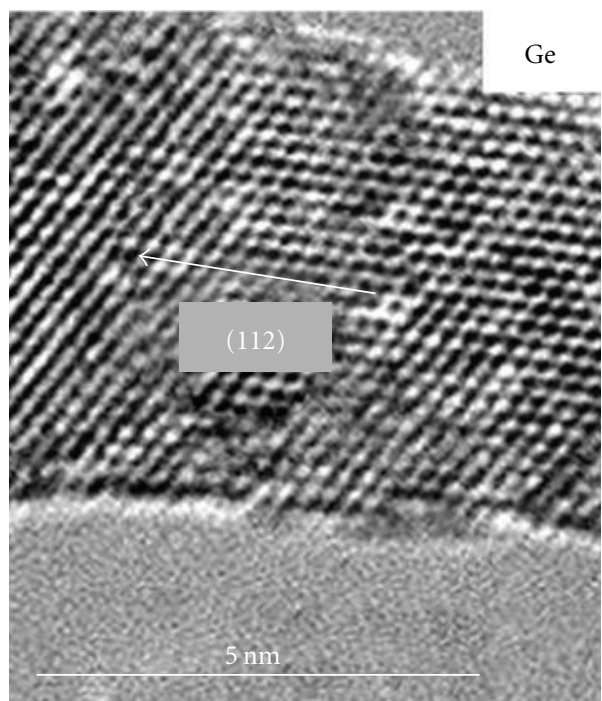

(a)

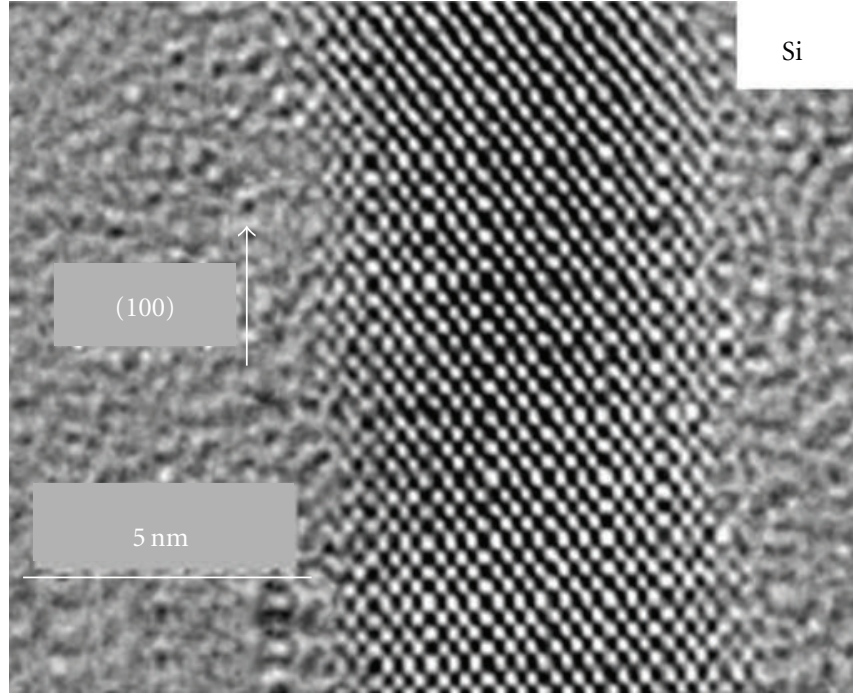

(b)

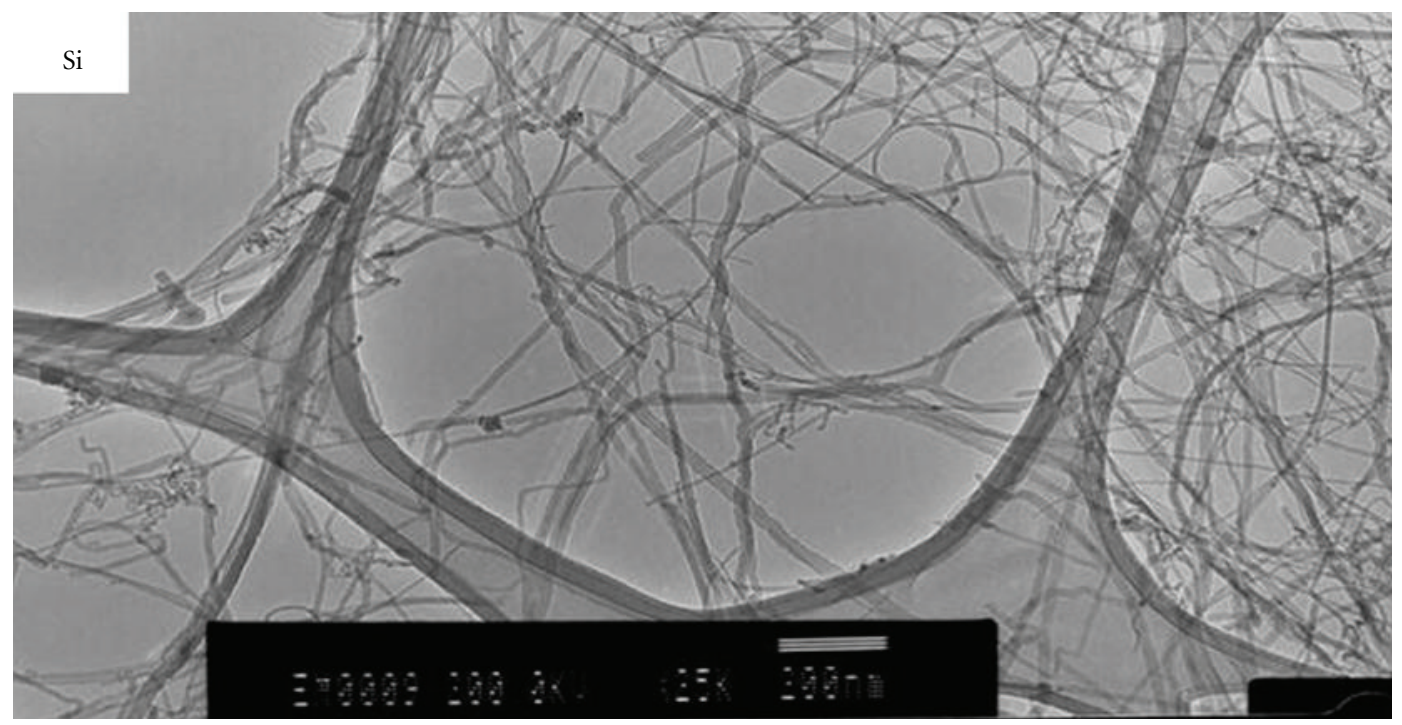

(c)

Figure 8: HRTEM images of (a) $6 \mathrm{~nm}$ diameter Ge nanowire [80], (b) $7 \mathrm{~nm}$ Si nanowire [47], and (c) LRTEM images of Si nanowires.

diameter distribution. There is a clear difference in the Raman profile of with and without the inclusion of the diameter distribution that demonstrates its importance in the interpretation of Raman spectra of confined phonons. It must be emphasized that the model is inconsistent at diameters $\leq 3 \mathrm{~nm}$. This has been discussed in detail in [44-47].

\section{Experimental Evidence of Confined Optical Phonons in Nanowires}

Optical phonons propagate in the lattice of a single crystal as a wave and exhibit dispersion depending on their wavelength, or equivalently their wavevector in the Brillouin zone [118]. The momentum conservation selection rule determines the region of the Brillouin zone that can be accessed in the Raman scattering process. In a Raman scattering experiment, the magnitude of the scattering vector is $2 k_{o} \sin (\theta / 2)$, where $k_{o}$ is the wavevector of the incident light and $\theta$ is the scattering angle. The maximum value of the scattering vector would be $2 k_{o}$ (in the backscattering configuration), $\sim 5 \times$ $10^{4} \mathrm{~cm}^{-1}$ for visible light. Hence, this wavevector is much smaller than the wavevector $q$ of the full phonon dispersion curve, which extends up to the boundary of the Brillouin zone $\left(\pi / a \sim 10^{8} \mathrm{~cm}^{-1}\right)$. Since acoustic phonons mostly have very small wavenumbers, they could only be probed with Brillouin spectrometer consisting of a Fabry-Perot interferometer. Thus, only the optical phonons close to the zone centre $(q \approx 0)$ are probed in visible Raman spectroscopy of bulk samples. This $q \approx 0$ selection rule is essentially a consequence of the infinite periodicity of the crystal lattice. However, if the periodicity of the crystal is curtailed, as in the case 


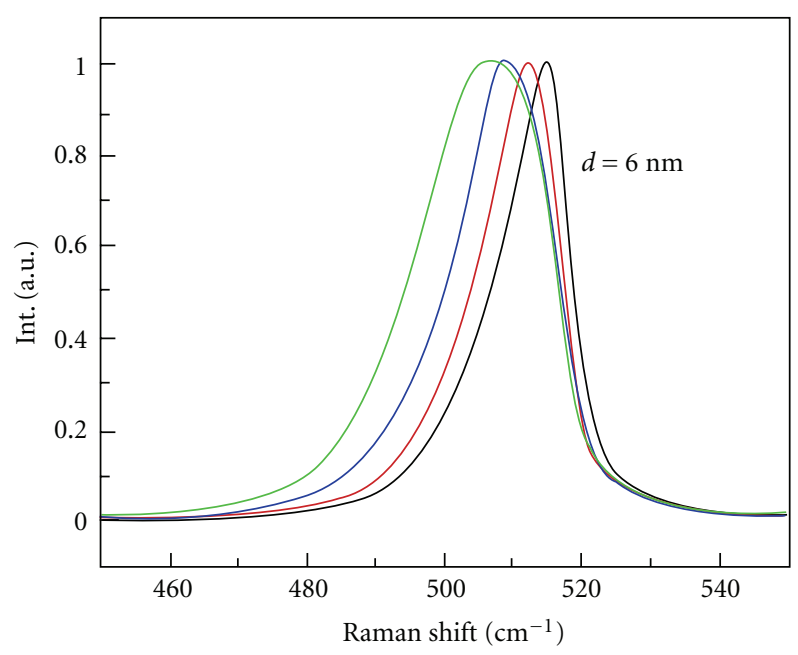

(a)



(b)

FIGURE 9: Calculated Raman line shape of the $520 \mathrm{~cm}^{-1}$ band of Si nanowires subjected to laser inhomogeneous heating. The focal spot size is $1 \mu \mathrm{m}$ in the micro-Raman focal plane. Results are calculated using (10) at the different peak temperatures indicated for (a) $D=6.0 \mathrm{~nm}$ and (b) $D=23 \mathrm{~nm}$ nanowires [44-46].

of nanocrystalline materials, the $q \approx 0$ selection rule is relaxed, leading to asymmetric broadening and downshift in the Raman profile, in the case of Si and Ge.

Additionally, it has also been observed that, the supporting substrate plays an important role in Raman scattering experiment $[44,46,80,124]$. If the supporting substrate is a poor thermal conductor, the Raman peak shift to lower frequency due to heating $[44,46,80]$. There is substantial evidence showing that both quantum confinement and inhomogeneous heating induce redshift and asymmetric broadening relative to the bulk, in Raman spectra of optical phonons in $\mathrm{Si}$ and Ge nanowires [44, 46, 122, 123, 125]. Here, we focus on phonon confinement of the first-order Raman spectrum in semiconducting nanowires of $\mathrm{Si}$ and $\mathrm{Ge}$ and would discuss the effect of inhomogeneous heating later.

3.1. Semiconducting Nanowires: Si and Ge. Si nanowire has always been the prototype for nanoscale semiconducting studies due to the vast information on its bulk counterpart. The earliest reports of phonon confinement in nanowire $[106,107]$ focused on Si. The shape and frequency of the Raman peaks due to scattering by the optical phonons are thought to be dependent on the diameter of the nanowire. The onset of phonon confinement is at the point where the phonon mean free path is comparable to the diameter of the nanowire [47]. Figure 7(a) shows the first systematic report on the evolution of Raman spectrum of Si nanowires with diameters for $3 \mathrm{~nm} \leq \mathrm{D} \leq 20 \mathrm{~nm}$. The open circles represent the raw Raman data, and the solid line is a least square fit of the Richter model including diameter distribution (3). It depicts an asymmetric broadening to lower frequency and a downshift as reported by Adu and coworkers [47]. Similar arguments can be made for Ge nanowires which have been reported by Jalilian and coworkers [80].

Figures 7(b) and 7(c) are the plots of measured TO optical phonon peak frequency, versus $\mathrm{Si}$ and Ge nanowire diameter $[47,80]$. The solid line is a guide to the eye. These plots show a significant difference between Ge and Si TO phonons. In the absolute shift case, the Si slope between 4.5 and $9.5 \mathrm{~nm}$ is $0.9086 \mathrm{~cm}^{-1} / \mathrm{nm}$ and the Ge slope between 5 and $10 \mathrm{~nm}$ is $0.7634 \mathrm{~cm}^{-1} / \mathrm{nm}$. As the nanowire diameter decreases, the absolute Raman shift of the TO phonon increases but the Raman shift relative to the bulk decreases, in accordance with the theory [47]. The Ge nanowire TO phonon apparently only reaches bulk-like behavior at a diameter between 10 and $20 \mathrm{~nm}$ whereas for silicon this occurs between 5 and $10 \mathrm{~nm}$. Both Ge and Si nanowires exhibit TO phonon confinement that induces an absolute Raman shift over a range of $5 \mathrm{~cm}^{-1}$ $[47,80]$. The ranges of Raman shift relative to bulk, however, are approximately 2.6 and $7 \mathrm{~cm}^{-1}$ for $\mathrm{Si}$ and Ge, respectively. Although the absolute Raman shift of both Si and Ge shows positive curvature with nanowire diameter, the curvature is negative for $\mathrm{Ge}$ in the relative shift. It should be noted that the excitation laser wavelength used in Figures 7(b) and 7(c) is nearly in resonance with the Si sample but far from it for the Ge sample.

\section{Effect of Inhomogeneous Laser Heating on Confined Optical Phonons}

Typically, the laser irradiation during Raman measurements can cause sample heating and photon excitation of charge carriers [104, 126-130]. Downshifting and broadening of Raman bands in bulk materials, with increasing laser radiation, is typically identified to be due to laser heating. Poor 


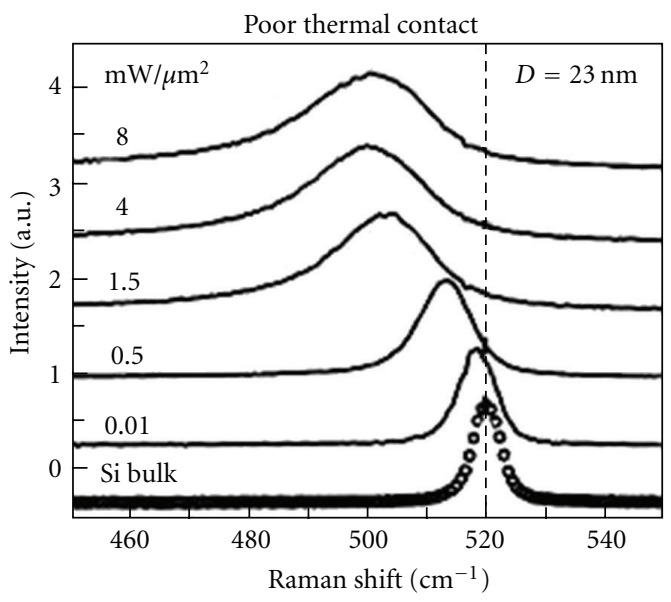

(a)

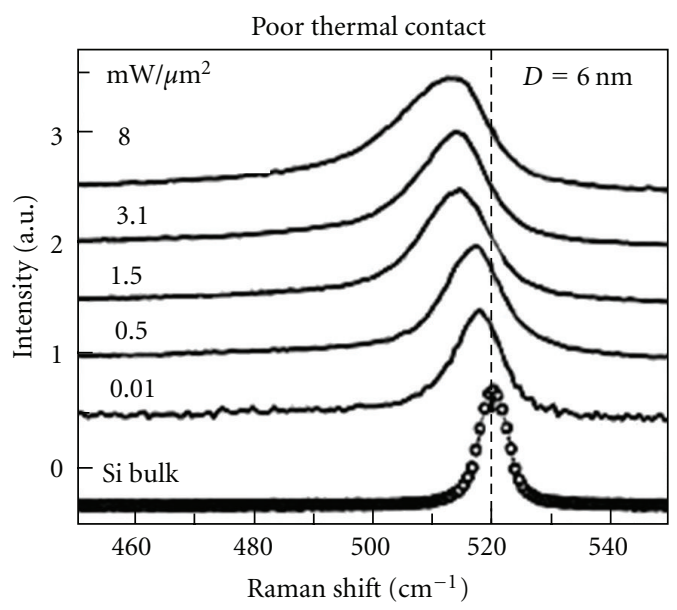

(c)



- Poor thermal contact

$\square$ Good thermal contact

(e)

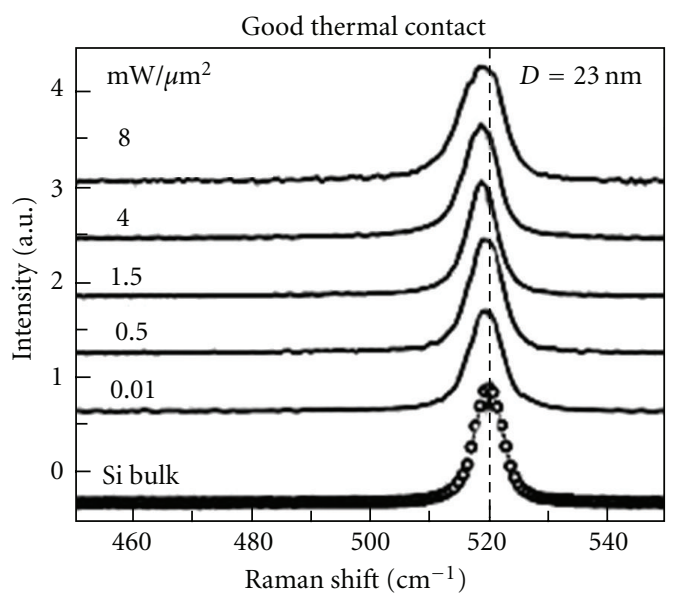

(b)

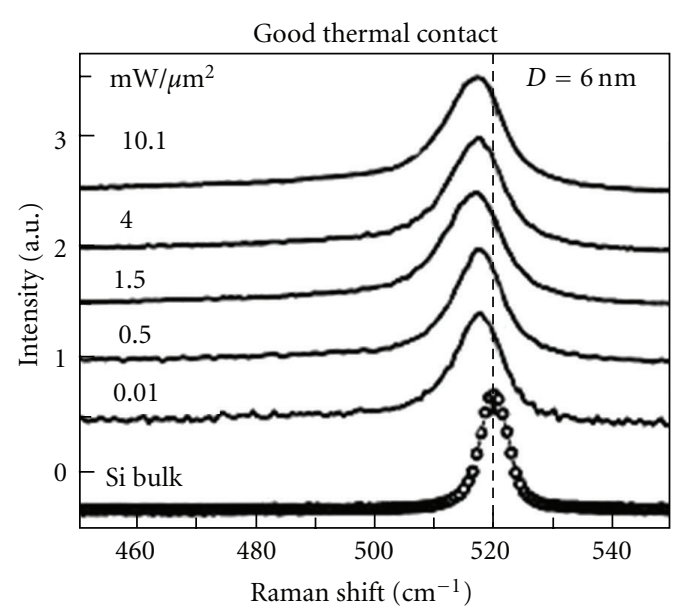

(d)

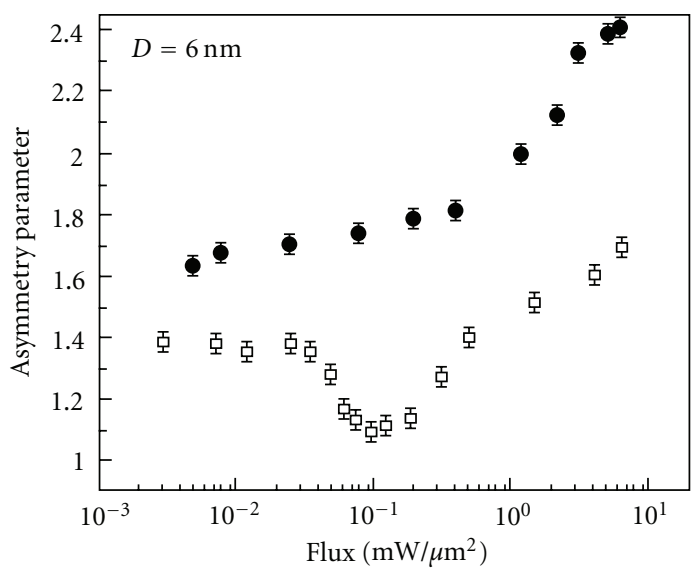

- Poor thermal contact

Good thermal contact

(f)

FigurE 10: Flux $(\Phi)$ dependent Raman spectra of Si nanowires collected under poor thermal anchorage (a, c) and good thermal anchorage $(\mathrm{b}, \mathrm{d})$ for $23 \mathrm{~nm}$ wires $(\mathrm{a}, \mathrm{b})$ and $6.0 \mathrm{~nm}$ wire (c, d). The nanowires were located on an Indium substrate. (b) Flux $(\Phi)$ dependence of the asymmetry parameter A for Si nanowires under poor thermal contact (solid circles) and good thermal contact (open squares) for (e) $23 \mathrm{~nm}$ (f) $6.0 \mathrm{~nm}$ Si nanowires [46]. 


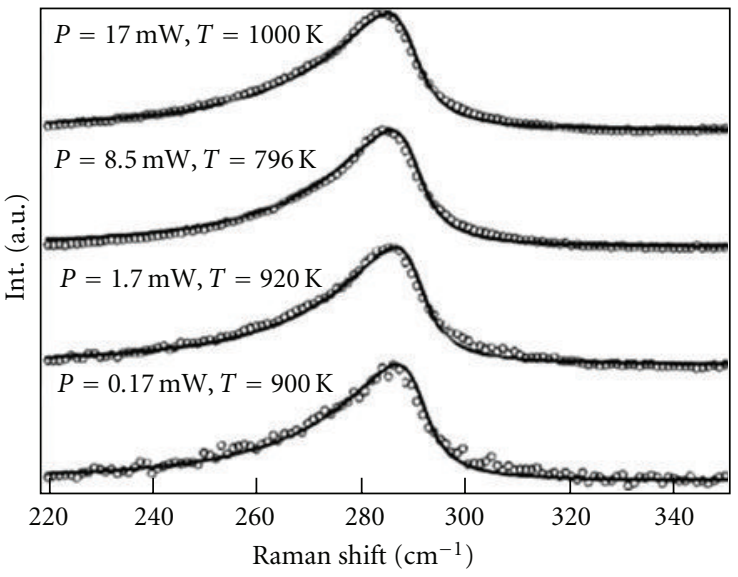

a



5

(a)

(b)

FIGURE 11: Raman spectra at $632.8 \mathrm{~nm}$ for the Ge nanowire $(D=7 \mathrm{~nm})$ for varying laser power levels (circles) at two different Ga droplet sizes (a) droplet size $\sim 1 \mu \mathrm{m}$ and (b) droplet size $\sim 1 \mathrm{~mm}$. The solid lines represent the least square fit (10) [80].

thermal contact of the nanowire to the substrate and intense focusing of the laser beam in a micro Raman spectrometer can lead to significant heating of the sample. In this section, we discuss the temperature dependence of the first-order Raman spectra of Ge and Si nanowire due to variation in laser power and thermal conductance of the nanowire on the substrate. The subtle interplay between quantum phonon confinement and the local heating effect are shown to influence both the frequency shift and the asymmetric broadening of the Raman spectra.

The Stokes-anti-Stokes intensity ratio of first-order Raman spectra is normally used to determine the local temperature of the nanowire sample. The intensity ratio relates to the temperature by

$$
\frac{I_{\mathrm{s}}}{I_{\mathrm{as}}}=P_{o} \exp \left(\frac{\hbar \omega_{o}}{k_{B} T}\right),
$$

where $\omega_{o}$ is the phonon frequency, the prefactor $P_{o}$ depends on the absorption constant and the Raman cross-section at a given frequency. Typically, the value of $P_{o}$ is determined by calculating the Stokes-anti-Stokes intensity ratio $I_{\mathrm{S}} / I_{\mathrm{as}}$ at room temperature and at the lowest possible laser power to eliminate any laser-induced heating. The phonon confinement function (3) is modified to include temperature dependence [128-130]. The frequency is expressed as

$$
\omega(q, T)=\omega(q)+\Delta \omega_{1}(T)+\Delta \omega_{2}(T)
$$

$\Delta \omega_{1}(T)$, is the frequency shift due to phonon decay processes. This phonon-phonon coupling term describes the anharmonic coupling between phonons and is approximated by

$$
\Delta \omega_{1}(T)=A_{1}\left(1+\frac{2}{x}\right)+A_{2}\left[1+\frac{3}{x}+\left(1+\frac{3}{x^{2}}\right)\right],
$$

where $x=e^{\left(\hbar \omega_{0} / 2 \kappa_{B} T\right)}-1$. The first term describes the coupling of the phonon to decayed low-energy two phonons (three-phonon coupling), and the second term describes the coupling to three decayed phonons (four-phonon coupling). $A_{1}$ is adjusted to match the bulk Ge and Si values. $\Delta \omega_{2}(T)$ is the frequency shift due to the thermal expansion of the lattice. The compressive stress expressed as

$$
\Delta \omega_{2}(T)=\omega_{o}\{\exp (3 \gamma \beta T)-1\}
$$

is neglected since the Si and Ge nanowires used in the studies exhibit limited or no oxide sheath formation as evidenced by Figure 8. $\gamma$ is the Gruneisen parameter, and $\beta$ is the thermal expansion coefficient of Ge or Si.

The temperature dependence of the inverse phonon life time (FWHM) in the formalism [128-130] is

$$
\Gamma(T)=\Gamma+\Delta \Gamma(T),
$$

where $\Gamma$ is the FWHM of the bulk Ge or Si including the instrumental broadening, and $\Delta \Gamma$ is given as

$$
\Delta \Gamma(T)=B_{1}\left(1+\frac{2}{x}\right)+B_{2}\left[1+\frac{3}{x}+\left(1+\frac{3}{x^{2}}\right)\right],
$$

where $x$ is as defined previously. The first term is due to the three-phonon coupling, and the second term is the result of the four-phonon coupling effect on the FWHM of the Raman spectrum. Just as before, we neglect the four-phonon coupling term, and $B_{1}$ is adjusted to match the bulk Ge or Si values.

The asymmetric broadening of the Raman TO band has been attributed to Fano interference between scattering from the discrete optical phonon and an electronic continuum due to photon-excited charge carriers [126]. At sufficient doping levels, electrons and holes in semiconductors participate in inelastic scattering leading to Fano resonance. An asymmetry of the Fano line shape depends on the electron-phonon coupling strength. Attributable to the observation of asymmetry in the low-frequency tail, the contribution of Fano 


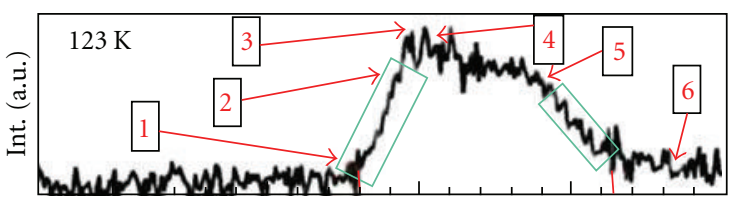

(a)



(b)

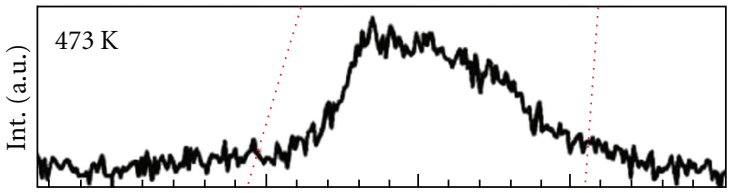

(c)

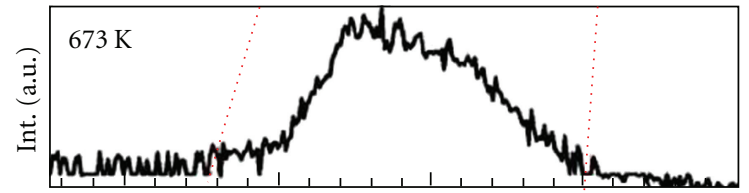

(d)

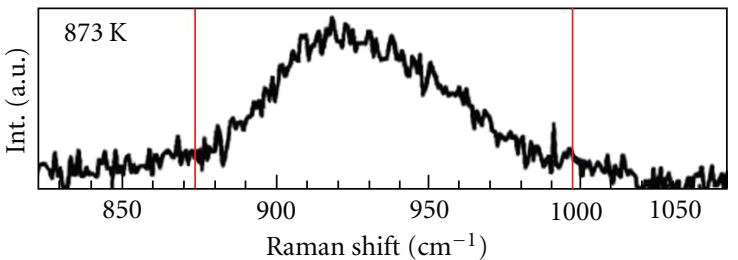

(e)

Figure 12: Second-order Raman spectra of Si nanowires TO phonons as a function of temperature. The numbers in the top panel $(1,2,3,4,5$, and 6) refer to Brillouin zone points $\mathbf{X}, \mathbf{Q}$, $\mathrm{S}_{1}, \mathrm{~W}, \mathrm{~L}$, and $\Gamma$, respectively. Temperature-dependent second-order Raman spectra of Si obtained using $632.8 \mathrm{~nm}$ laser excitation [113].

resonance for asymmetric broadening of the Raman spectrum can be ruled out, since laser excitations generate equal numbers of holes and electrons in the undoped Ge and Si. The asymmetric line shape in the phonon mode is also known to be affected by strain caused by an oxide layer. There were no indications that the limited oxide layer had any contribution to the asymmetric line shape.

A coupled phenomenological model that accounts for both phonon confinement and temperature effects to best describe the observed asymmetry in the first-order Raman spectra for Ge and Si nanowires is expressed as

$$
\begin{aligned}
& I(\omega, D, T) \\
& \quad=\int_{0}^{1} \int_{0}^{\infty} \frac{e^{-\left(q_{\perp}^{2} D^{2} / 4 \alpha^{2}\right)} e^{\left[(D-\bar{D})^{2} / 2 \sigma^{2}\right]}}{\left[\omega-\omega\left(q_{\perp}, T\right)+\Delta \omega\right]^{2}+(\Gamma+\Delta \Gamma / 2)^{2}} 2 \pi d q_{\perp} d D,
\end{aligned}
$$

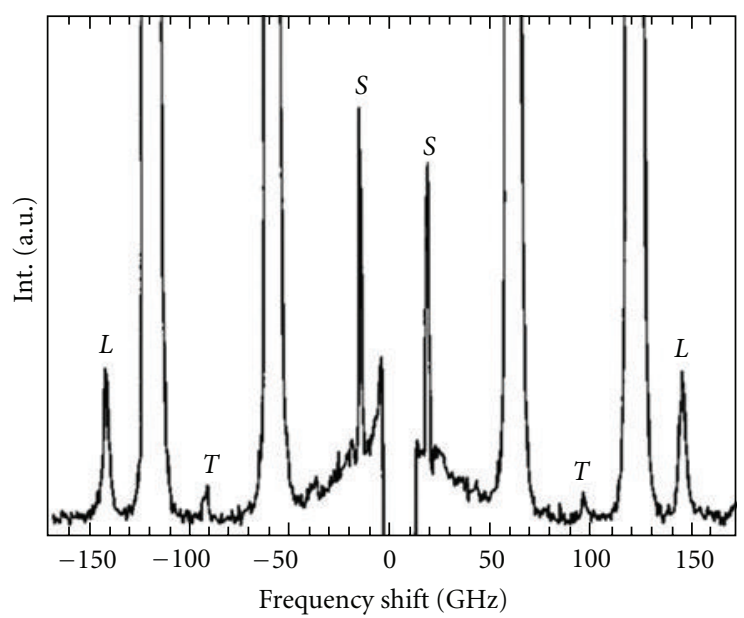

Figure 13: The Brillouin spectrum of bulk Si showing a broad quasielastic peak around the central elastically scattered peak. The peaks labeled $(S),(T)$, and $(L)$ correspond to the surface acoustic wave, transverse mode $(T)$, and longitudinal mode $(L)$, respectively [114].

where $e^{\left[(D-\bar{D})^{2} / 2 \sigma^{2}\right]}$ is the diameter distribution (log-normal) of the nanowires. Equation (10) shows that the coupled phonon confinement and inhomogeneous local laser heating induce a redshift and asymmetric broadening in the Raman spectra of Si and Ge nanowires. Figure 9 shows the evolution of phonon line shape with temperature of $6 \mathrm{~nm}$ and $23 \mathrm{~nm} \mathrm{Si}$ nanowire calculated using (10).

\section{Experimental Evidence of Inhomogeneous Laser Heating in Si and Ge Nanowires}

A number of reports on inhomogeneous laser heating effect in the first-order Raman spectrum of nanowires have been published in the literature $[44-46,80,104,110,113,130$ 132]. Here, we summarize our results on the coupled phonon confinement and inhomogeneous laser heating in Si and Ge nanowires. Figure 10 shows the Raman spectra of ensemble of Si nanowires with most probable diameter of $6 \mathrm{~nm}$ and $23 \mathrm{~nm}$ under good $(\mathrm{b}, \mathrm{d})$ and poor $(\mathrm{a}, \mathrm{c})$ thermal anchoring to the supporting substrate $[44,46]$. Both $6 \mathrm{~nm}$ and $23 \mathrm{~nm}$ diameter nanowires show substantial redshift with increasing flux when they are inefficiently coupled thermally to the supporting substrate. For $23 \mathrm{~nm}$ diameter nanowires, there is very little frequency difference between high and low flux when there is good thermal contact between the nanowires and the supporting substrate. In the case of the $6 \mathrm{~nm}$ diameter Si nanowires which are poorly anchored to the substrate, we see a redshift in the peak position as a function of increasing flux. The corresponding plot of the asymmetry as a function of laser flux is shown in Figures $10(\mathrm{e})$ and 10(f). The $23 \mathrm{~nm}$ nanowires exhibit monotonic increase in asymmetry with increasing laser flux for both poor and good thermal anchorage to the supporting substrate (Figure 10(e)). On the other hand, for the $6 \mathrm{~nm}$ Si nanowires with good thermal anchorage to the substrate, the asymmetry initially remains flat, then decreases, and eventually increases with increasing 


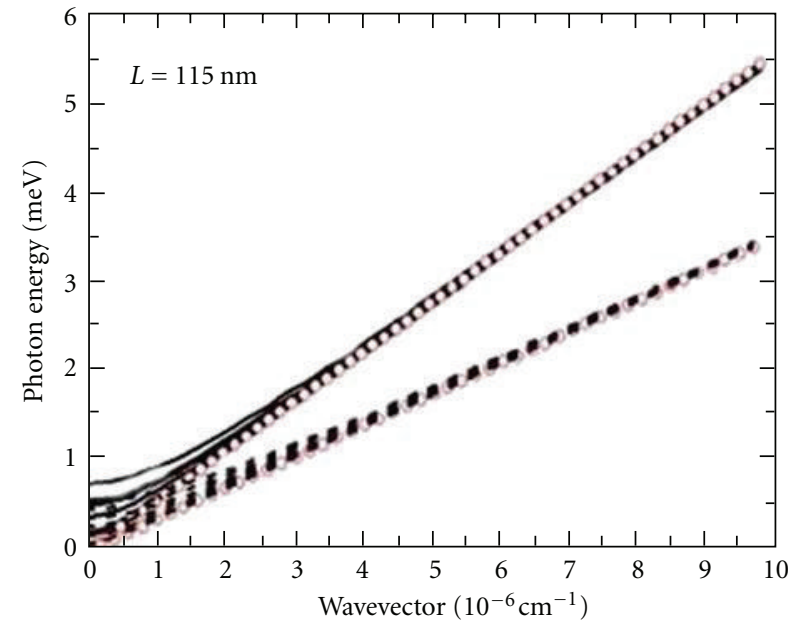

(a)



- NW longitudinal modes

- - NW transversal modes

○ Bulk modes



(b)

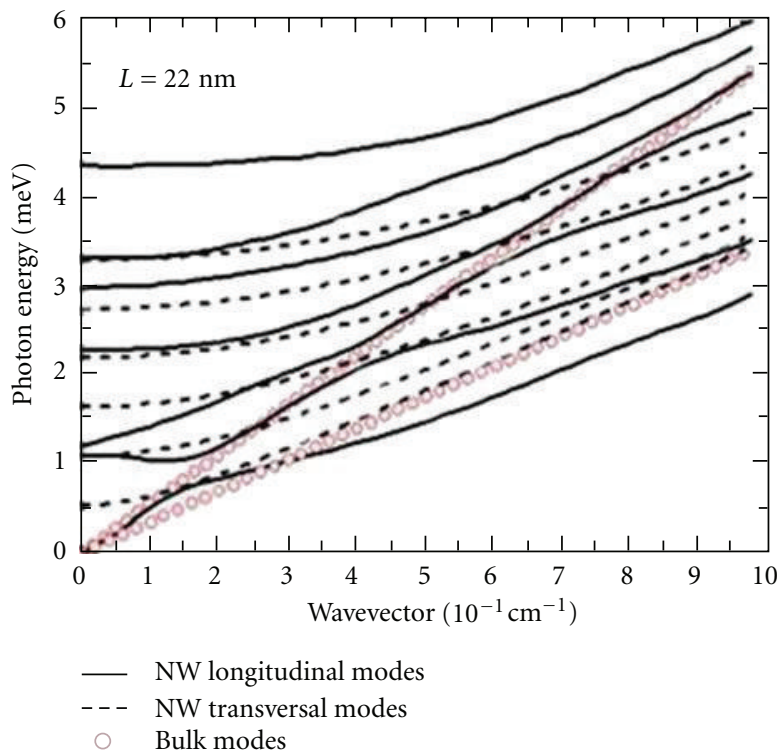

(d)

FIGURE 14: Elastic continuum approximation prediction of the dispersion relations for longitudinal (solid lines) and transversal (dashed lines) modes in Si nanowires of cross-sections 115, 56, 37, and $22 \mathrm{~nm}$ [94].

flux. The nonmonotonicity observed in the asymmetry of the $6 \mathrm{~nm}$ nanowires is attributed to low coverage and good thermal contact with the Indium substrate which clearly demonstrates the interplay of phonon confinement (dominant at low flux) and inhomogeneous laser heating (dominant high flux) $[44,46]$. Similarly, the Raman spectrum of Ge nanowires on quartz and germanium substrates exhibited analogous characteristics as reported by Jalilian et al. [80]. The heating effect is found to be more pronounced for nanowires grown and analyzed on quartz (thermal conductivity $\kappa \sim$ $6 \mathrm{w} / \mathrm{mK})$ than for those grown on $\mathrm{Ge}(\kappa \sim 60 \mathrm{~W} / \mathrm{mK})$ substrates for a given laser power as shown in Figure 11.

Effects of laser heating have also been observed in second-order Raman spectra of Si nanowires as shown in Figure 12 [113]. The temperature-induced Raman redshift of the $2 \mathrm{TA}(\mathbf{X})$ and $2 \mathrm{TO}$ peaks of Si nanowires were enhanced compared with those of bulk Si. The Raman shift of the $2 \mathrm{TO}$ phonon peak of Si nanowires is larger, and thus it is expected that the $2 \mathrm{TO}(\boldsymbol{\Gamma})$ temperature dependence is weaker than the temperature dependence of bulk Si. Additionally, at $300 \mathrm{~K}$, the frequency of $2 \mathrm{TO}(\boldsymbol{\Gamma})$ of Si nanowires (bulk silicon) is $1041 \mathrm{~cm}^{-1}\left(1037 \mathrm{~cm}^{-1}\right)$ [113]. An earlier study of the same effects provided analytical inclusion of the anharmonic phonon processes. The asymmetry of Raman peaks was ascribed to quantum confinement since heating alone produces symmetric broadening. The integrated intensity of the phonon modes as a function of measurement temperatures has been reported. One of the earliest studies of size effects in secondorder Si mode identified the transition as originating in the Brillouin zone $\mathbf{L}$ point. The validity of assigning quantum confinement effects to overtone transitions has been questioned without explanation [133]. 


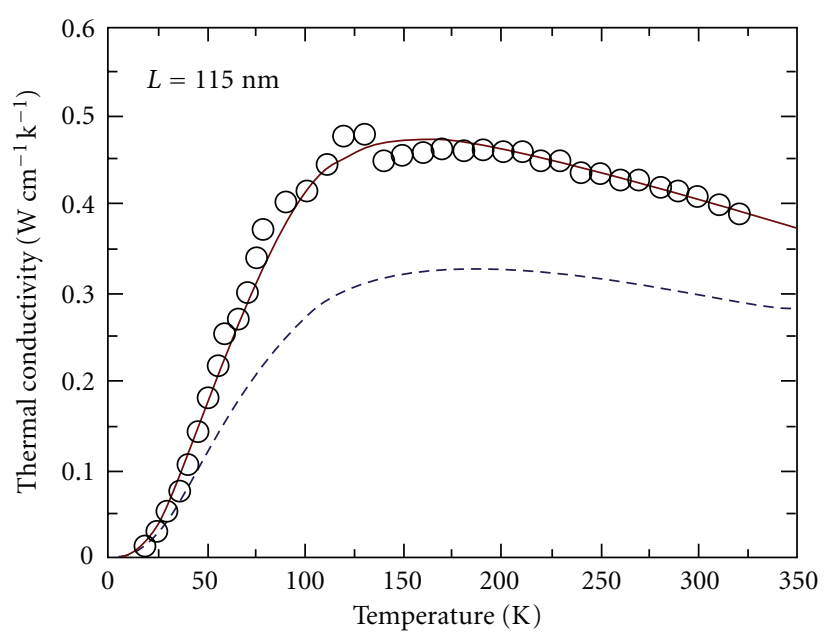

(a)

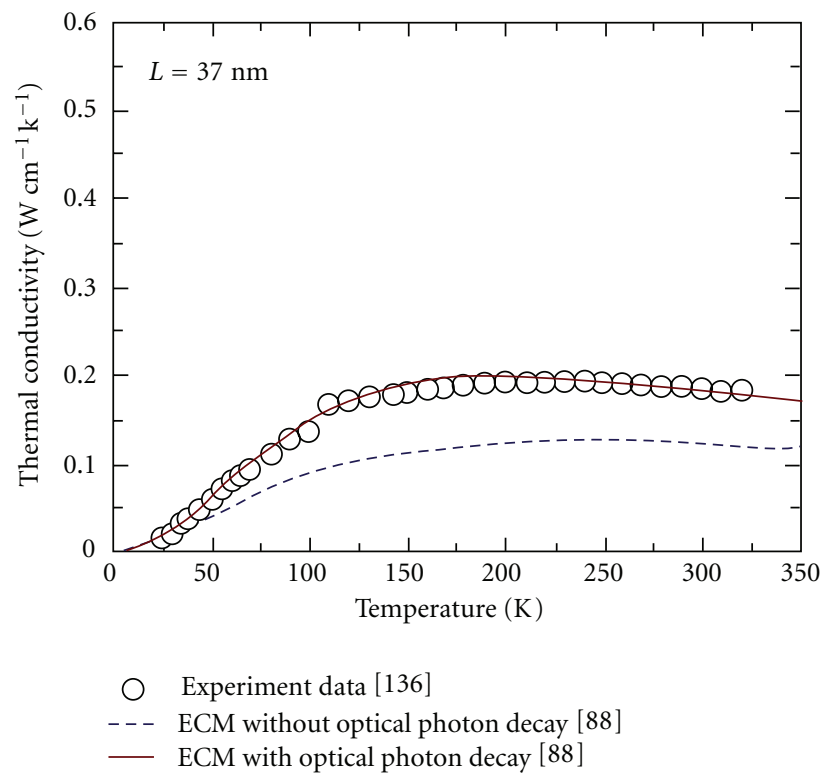

(c)

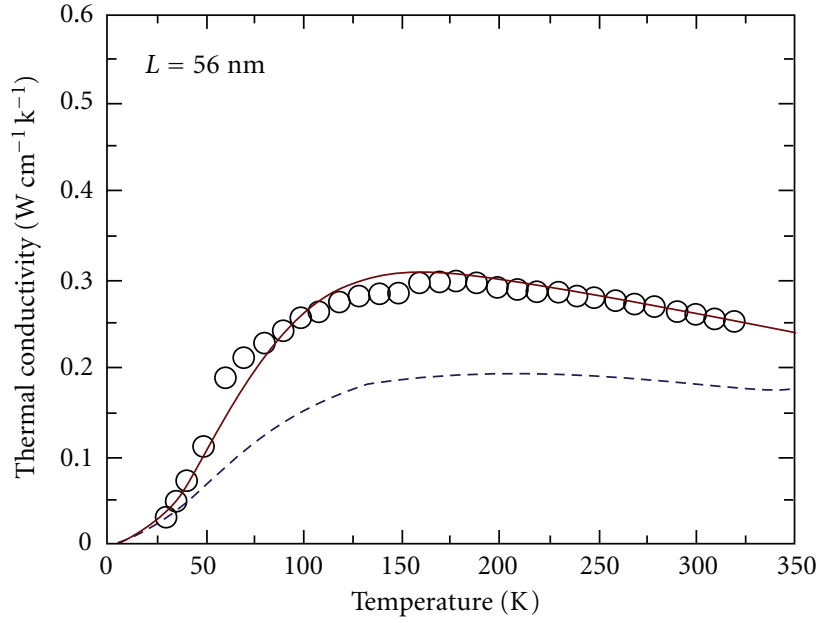

(b)

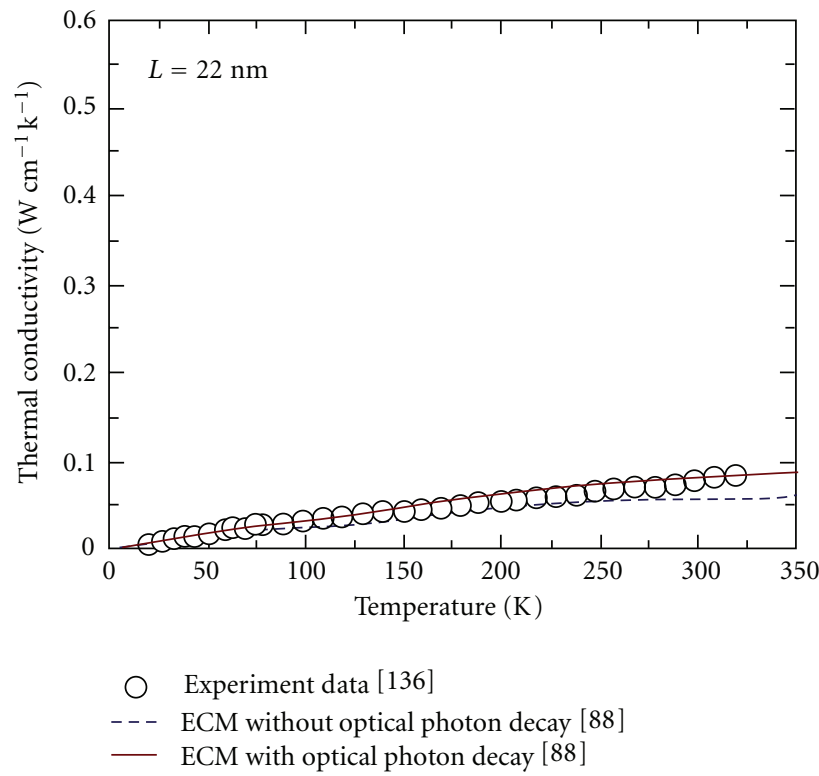

(d)

FIGURE 15: Thermal conductivity of individual Si nanowire. Symbols: experimental data [115]. Solid lines: elastic continuous model (ECM) which considers the optical phonons decay into acoustic phonons as acoustic phonon generation rate. Dashed lines: theoretical results obtained from ECM without accounting for the optical phonon decay.

\section{Acoustic Phonon Confinement}

Since the total wavevector in an inelastic light scattering process is conserved, that is, $\Delta(k+q)=0$ (where $k$ and $q$ are the wavevectors of the photons and phonons, resp.), the wavevector of resulting phonon in a backscattering configuration is $q=2 n k_{i}$, where $k_{i}$ is photon wavevector and $n$ is the refractive index of the solid. In acoustic phonons, this "selection rule" predicts a narrow peak at a frequency shift $\omega=v_{s} q=2 v_{s} n k_{i}$, where $v_{s}$ is the phonon sound velocity. For $\mathrm{Si}$, these peaks are very close to the laser line, normally observed within the range $0.7-7 \mathrm{~cm}^{-1}(\sim 20-200 \mathrm{GHz})$. Brillouin scattering technique is used to detect such lowfrequency excitations. Shown in Figure 13 is Brillouin spectrum of bulk Si [114]. The sharp peaks labeled $L, T$, and $S$ are stokes/antistokes pair of the longitudinal $(L)$, transverse $(T)$ and surface acoustic waves $(S)$, respectively. Note that the unit of the frequency is in $\mathrm{GHz}$.

The acoustic phonon dispersion of nanowires is more complex and significantly altered compared to their bulk counterpart. As shown in Figure 2(c) for bulk Si, there is one $L A$ and two TA modes. In nanowires, however, the number of acoustic phonon modes is influenced by the nanowire diameter. Shown in Figure 14 is the phonon dispersion of the seven lowest acoustic branches in Si nanowire of diameter $115,56,37$, and $22 \mathrm{~nm}$ calculated using the elastic continuum model (ECM) [94], together with the three branches of the bulk. It can be inferred quantitatively that the slope of the acoustic phonon branches decreases with decreasing 


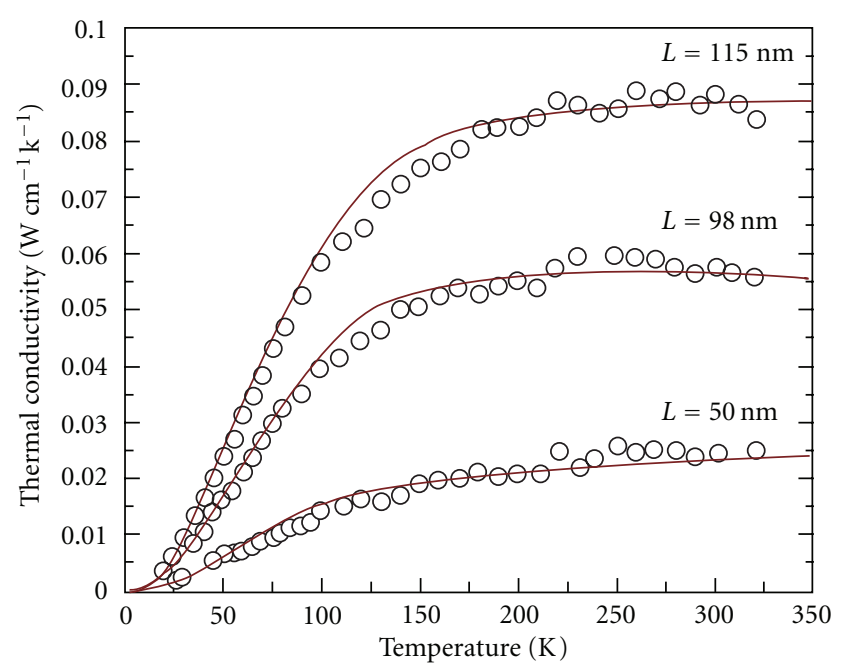

O Experiment data [136]

_ EMC with optical photon decay [88]

FIGURE 16: Thermal conductivity of rough Si nanowires. Open circles are experimental results from [116], and solid lines are theoretical results obtained from the elastic continuum model including acoustic phonon confinement and surface roughness [94].

diameter of the nanowires, which implies a decrease in the transverse and longitudinal average phonon velocities. To date, there are no reports on Brillouin scattering on Si and Ge nanowires. Recently, Torres and coworkers observed acoustic phonon confinement in Si membranes of $32.5 \mathrm{~nm}$ thickness by suspending the membranes over a trench to prevent damping [134].

6.1. Thermal Conductivity. Extensive work on the effects of acoustic phonon confinement in III-V compounds and Sibased nanostructures shows a lower acoustic phonon group velocity, lower thermal conductivity, and increased scattering rate compared to their bulk counterpart [135-137]. The acoustic phonon scattering rate is affected by the nanowire surface roughness and the confinement in the circumferential direction (when the diameter is comparable to the phonon mean free path).

Generally, the thermal conductivity $(k)$ can be expressed in terms of heat capacity $(C)$, the phonon mean free path $(l)$, and the acoustic phonon group velocity $(v)$ as

$$
\kappa=\frac{1}{3} v C l
$$

Thus, the reduction of the acoustic phonon average velocity and the scattering effect cause the thermal conductivity of nanowire to decrease with decreasing wire diameter. Figure 15 shows the experimental data-open circles [115] and theoretical fit using the (ECM) solid line [94] of thermal conductivity of $115 \mathrm{~nm}, 56 \mathrm{~nm}, 37 \mathrm{~nm}$, and $22 \mathrm{~nm} \mathrm{Si}$ nanowire with smooth surfaces. The fit takes into account the decay of the optical phonons into acoustic phonons. The calculation without the decay is shown as dashed line. Similar experiment on Si nanowire with surface roughness found an order of magnitude lower thermal conductivity, as shown in Figure 16 [116]. These reports clearly show the potential of using phonon engineering techniques such as surface roughness in addition to core-shell techniques to nanoengineer nanowires for applications where low thermal conductivity and high electron conduction (mobility) are highly desirable, such as thermoelectric nano coolers.

\section{Summary and Conclusions}

In this paper, we reviewed phonon confinement effects in nanowire using Si and Ge-nanowire as prototypes. The concepts reviewed here are applicable to nanowires of semiconducting materials. We presented both experimental and theoretical models, first proposed by Richter and coworkers that explain the experimentally observed phonon confinement (downshift and asymmetric broadening) effect in Si and Ge nanowires and the interplay of phonon confinement and inhomogeneous laser heating in the Raman spectra of the first-order Raman bands of smaller Si $(D \leq 10 \mathrm{~nm})$ and Ge $(D \leq 15)$ nanowires. Thus, to appreciate the phonon confinement effect in Raman spectroscopy of semiconducting nanowire, it is critical and necessary to use ultra low laser flux and high thermal conductivity substrates with good thermal anchoring.

We also presented the influence of the radial confinement in nanowires on acoustic phonons, particularly in $\mathrm{Si}$ nanowires. Theoretical calculations using elastic continuummodel predicted the number of acoustic branches being greater than or equal to four depending on the nanowire diameter. For Si nanowires, both theoretical calculations and experimental measurements clearly revealed suppression of thermal conductivity of nanowires with decreasing nanowire diameter. The suppression is attributed to acoustic phonon confinement and nanowire surface roughness.

To date, there have been limited reports on experimental measurements of acoustic phonons in nanowires, especially Si and Ge. Sensitive experiments are needed to probe these new phonon properties in "confined" nanowires.

\section{Acknowledgments}

This work was supported, in part, by funding from NSF (ECCS-0925835) and by Penn State Altoona Research and Sponsored Programs.

\section{References}

[1] C. V. Raman and K. S. Krishnan, "A new type of secondary radiation," Nature, vol. 121, no. 3048, pp. 501-502, 1928.

[2] T. H. Maiman, "Stimulated optical radiation in Ruby," $\mathrm{Na}$ ture, vol. 187, no. 4736, pp. 493-494, 1960.

[3] T. H. Maiman, "Optical and microwave-optical experiments in Ruby," Physical Review Letters, vol. 4, no. 11, pp. 564-566, 1960.

[4] T. H. Maiman, "Stimulated optical emission in Ruby," Journal of the Optical Society of America, vol. 50, no. 11, p. 1134, 1960.

[5] T. N. Dung, D. J. Kim, and K. S. Kim, "Controlled synthesis and biomolecular probe application of gold nanoparticles," Micron, vol. 42, no. 3, pp. 207-227, 2011. 
[6] J. Xu, L. Zhang, H. Gong, J. Homola, and Q. Yu, "Tailoring plasmonic nanostructures for optimal SERS sensing of small molecules and large microorganisms," Small, vol. 7, no. 3, pp. 371-376, 2011.

[7] E. Spain, R. Kojima, R. B. Kaner et al., "High sensitivity DNA detection using gold nanoparticle functionalised polyaniline nanofibres," Biosensors and Bioelectronics, vol. 26, no. 5, pp. 2613-2618, 2011.

[8] W. Xie, P. Qiu, and C. Mao, "Bio-imaging, detection and analysis by using nanostructures as SERS substrates," Journal of Materials Chemistry, vol. 21, no. 14, pp. 5190-5202, 2011.

[9] I. Lee, X. Luo, X. T. Cui, and M. Yun, "Highly sensitive single polyaniline nanowire biosensor for the detection of immunoglobulin G and myoglobin," Biosensors and Bioelectronics, vol. 26, no. 7, pp. 3297-3302, 2011.

[10] S. Habouti, M. Mátéfi-Tempfli, C. -H. Solterbeck, M. EsSouni, S. Mátéfi-Tempfli, and M. Es-Souni, "Self-standing corrugated Ag and Au-nanorods for plasmonic applications," Journal of Materials Chemistry, vol. 21, no. 17, pp. 6269-6273, 2011.

[11] B. R. Wood, E. Bailo, M. A. Khiavi et al., "Tip-enhanced raman scattering (TERS) from hemozoin crystals within a sectioned erythrocyte," Nano Letters, vol. 11, no. 5, pp. 1868 1873, 2011.

[12] Z. V. Popović, Z. Dohčević-Mitrović, M. Śćepanović, M. Grujić-Brojčin, and S. Aŝkrabić, "Raman scattering on nanomaterials and nanostructures," Annalen der Physik, vol. 523, no. 1-2, pp. 62-74, 2011.

[13] T. Vo-Dinh, H. N. Wang, and J. Scaffidi, "Plasmonic nanoprobes for SERS biosensing and bioimaging," Journal of Biophotonics, vol. 3, no. 1-2, pp. 89-102, 2010.

[14] R. A. Alvarez-Puebla and L. M. Liz-Marzán, "SERS-based diagnosis and biodetection," Small, vol. 6, no. 5, pp. 604-610, 2010.

[15] M. P. Cecchini, J. Hong, C. Lim et al., "Ultrafast surface enhanced resonance raman scattering detection in dropletbased microfluidic systems," Analytical Chemistry, vol. 83, no. 8, pp. 3076-3081, 2011.

[16] R. Contreras-Cáceres, S. Abalde-Cela, P. Guardia-Girós et al., "Multifunctional microgel magnetic/optical traps for SERS ultradetection," Langmuir, vol. 27, no. 8, pp. 4520-4525, 2011.

[17] W. Kiefer (ed.), “Tip-enhanced Raman spectroscopy,” Journal of Raman Spectroscopy, vol. 40, no. 10, pp. 1335-1457, 2009.

[18] E. Kasper and K. Lyutovich, Eds., Properties of Silicon and Germanium and SiGe: C, INSPEC, London, UK, 2000.

[19] G. Nilsson and G. Nelin, "Study of the homology between silicon and germanium by thermal-neutron spectrometry," Physical Review B, vol. 6, no. 10, pp. 3777-3786, 1972.

[20] W. Jian, Z. Kaiming, and X. Xide, "Reinvestigation of the TA modes in Ge and Si in Born-von Kármán model," Solid State Communications, vol. 86, no. 11, pp. 731-734, 1993.

[21] G. Dolling, Inelastic Scattering of Neutrons in Solids and Liquids, International Atomic Energy Agency, Viena, Austria, 1963.

[22] G. Nilsson and G. Nelin, "Phonon dispersion relations in Ge at 80 K," Physical Review B, vol. 3, no. 2, pp. 364-369, 1971.

[23] J. E. Smith Jr., M. H. Brodsky, B. L. Crowder, and M. I. Nathan, "Raman scattering in amorphous $\mathrm{Si}, \mathrm{Ge}$ and III-V semiconductors," Journal of Non-Crystalline Solids, vol. 8-10, pp. 179-184, 1972.

[24] R. Beserman and T. Bernstein, "Raman scattering measurement of the free-carrier concentration and of the impurity location in boron-implanted silicon," Journal of Applied Physics, vol. 48, no. 4, pp. 1548-1550, 1977.
[25] J. Ibáñez, R. Cuscó, and L. Artús, "Raman scattering determination of free charge density using a modified hydrodynamical model," Physica Status Solidi B, vol. 223, no. 3, pp. 715-722, 2001.

[26] W. Limmer, J. Gerster, M. Aigle et al., "Spatially resolved characterization of material composition and free carrier concentration by micro-Raman spectroscopy at surface selectively grown layers," Journal of Crystal Growth, vol. 188, no. 1-4, pp. 225-230, 1998.

[27] B. Boudart, B. Prévot, and C. Schwab, "Free-carrier concentration in n-doped InP crystals determined by Raman scattering measurements," Applied Surface Science, vol. 50, no. 14, pp. 295-299, 1991.

[28] M. Park, J. J. Cuomo, B. J. Rodriguez, W. C. Yang, R. J. Nemanich, and O. Ambacher, "Micro-Raman study of electronic properties of inversion domains in GaN-based lateral polarity heterostructures," Journal of Applied Physics, vol. 93, no. 12, pp. 9542-9547, 2003.

[29] M. Gargouri, B. Prevot, and C. Schwab, "Raman scattering evaluation of lattice damage and electrical activity in beryllium-implanted gallium arsenide," Journal of Applied Physics, vol. 62, no. 9, pp. 3902-3911, 1987.

[30] J. B. Renucci, M. A. Renucci, and M. Cardona, "Raman sepctroscopy in Ge-Si alloys," in Proceedings of the International Conference on Light Scattering in Solids, Flammarion Sciences, Paris, France, 1971.

[31] F. La Via, S. Privitera, M. G. Grimaldi, E. Rimini, S. Quilici, and F. Meinardi, "Determination of C54 nucleation site density in narrow stripes by sheet resistance measurements and $\mu$-Raman spectroscopy," Microelectronic Engineering, vol. 50, no. 1-4, pp. 139-145, 2000.

[32] S. Sahli and D. M. Aslam, "Effect of postdeposition anneal on the resistivitity of p-type polycrystalline diamond films," Applied Physics Letters, vol. 69, no. 14, pp. 2051-2052, 1996.

[33] M. I. Alonso and K. Winer, "Raman spectra of c-Si1-xGex alloys," Physical Review B, vol. 39, no. 14, pp. 10056-10062, 1989.

[34] C. S. Nichols, M. F. Ross, and C. Y. Fong, "Vibrational properties of amorphous silicon in the frequency range 250-450 $\mathrm{cm}^{-1}$," Physical Review B, vol. 32, no. 2, pp. 1061-1067, 1985.

[35] S. Fujii, "Process and apparatus for Raman spectra detection of defects in anodized oxide films in semiconductor devices," Japanese Kokai Tokkyo Koho, p. 6, 2003.

[36] T. Noda, "Semiconductor analysis apparatus, semiconductor analysis method and method for manufacturing semiconductor device," US Patent, 2003.

[37] J. F. Morhange, R. Beserman, and J. Bourgoin, "The use of Raman scattering for defect study in ion implanted semiconductors," in Proceedings of the International Congress on Appllied Processus Electronic Ioniques, p. 171, 1974.

[38] D. D. Tuschel and J. P. Lavine, "Micro-Raman characterization of unusual defect structure in arsenic-implanted silicon," in Proceedings of the 1999 MRS Fall Meeting-Symposium P: Optical Microstructural Characterization of Semiconductors, 2000.

[39] A. D. Yoffe, "Low-dimensional systems: quantum size effects and electronic properties of semiconductor microcrystallites (zero-dimensional systems) and some quasi-two-dimensional systems," Advances in Physics, vol. 51, no. 2, pp. 799-890, 2002.

[40] A. D. Yoffe, "Semiconductor quantum dots and related systems: electronic, optical, luminescence and related properties of low dimensional systems," Advances in Physics, vol. 50, no. 1, pp. 1-208, 2001. 
[41] A. D. Yoffe, "Low-dimensional systems: quantum size effects and electronic properties of semiconductor microcrystallites (zero-dimensional systems) and some quasi-two-dimensional systems," Advances in Physics, vol. 42, no. 2, pp. 173-266, 1993.

[42] O. J. Pallotta, G. T. P. Saccone, and R. E. Woolford, "Biosensor system discriminating between the biliary and pancreatic ductal systems," Medical and Biological Engineering and Computing, vol. 44, no. 3, pp. 250-255, 2006.

[43] K. M. Sawicka, A. K. Prasad, and P. I. Gouma, "Metal oxide nanowires for use in chemical sensing applications," Sensor Letters, vol. 3, no. 1, pp. 31-35, 2005.

[44] K. W. Adu, H. R. Gutierrez, and P. C. Eklund, "Raman-active phonon line profiles in semiconducting nanowires," Vibrational Spectroscopy, vol. 42, no. 1, pp. 165-175, 2006.

[45] K. W. Adu, Q. Xiong, H. R. Gutierrez, G. Chen, and P. C. Eklund, "Raman scattering as a probe of phonon confinement and surface optical modes in semiconducting nanowires," Applied Physics A, vol. 85, no. 3, pp. 287-297, 2006.

[46] K. W. Adu, H. R. Gutiérrez, U. J. Kim, and P. C. Eklund, "Inhomogeneous laser heating and phonon confinement in silicon nanowires: a micro-Raman scattering study," Physical Review B, vol. 73, no. 15, Article ID 155333, 9 pages, 2006.

[47] K. W. Adu, H. R. Gutiérrez, U. J. Kim, G. U. Sumanasekera, and P. C. Eklund, "Confined phonons in Si nanowires," Nano Letters, vol. 5, no. 3, pp. 409-414, 2005.

[48] S. Bhattacharya, D. Banerjee, K. W. Adu, S. Samui, and S. Bhattacharyya, "Confinement in silicon nanowires: optical properties," Applied Physics Letters, vol. 85, no. 11, pp. 20082010, 2004.

[49] Y. Li, J. Xiang, F. Qian et al., "Dopant-free GaN/AlN/AlGaN radial nanowire heterostructures as high electron mobility transistors," Nano Letters, vol. 6, no. 7, pp. 1468-1473, 2006.

[50] F. Patolsky, G. Zheng, and C. M. Lieber, "Nanowire sensors for medicine and the life sciences," Nanomedicine, vol. 1, no. 1, pp. 51-65, 2006.

[51] F. Patolsky, G. Zheng, and C. M. Lieber, "Fabrication of silicon nanowire devices for ultrasensitive, label-free, realtime detection of biological and chemical species," Nature Protocols, vol. 1, no. 4, pp. 1711-1724, 2006.

[52] F. Patolsky, G. Zheng, and C. M. Lieber, "Nanowire-based biosensors," Analytical Chemistry, vol. 78, no. 13, pp. 42604269, 2006.

[53] G. F. Zheng, F. Patolsky, and C. M. Lieber, "INOR 32general and powerful platform for large-scale, label-free, parallel electrical detection of biomolecules by ultrasensitive nanowire transistor arrays," in Proceedings of the 231st ACS National Meeting, Atlanta, Ga, USA, March 2006.

[54] P. V. Radovanovic, C. J. Barrelet, S. Gradecak, F. Qian, and C. M. Lieber, "INOR 70-general synthesis and properties of manganese-doped II-VI and III-V diluted magnetic semiconductor nanowires," in Proceedings of the 230th ACS National Meeting, Washington, DC, USA, August 2005.

[55] Y. Li, F. Qian, J. Xiang, and C. M. Lieber, "Nanowire electronic and optoelectronic devices," Materials Today, vol. 9, no. 10, pp. 18-27, 2006.

[56] Y. Li, J. Xiang, F. Qian et al., "INOR 822-Nanowire radial heterostructures as high electron mobility transistors," in Proceedings of the 231st ACS National Meeting, Atlanta, Ga, USA, March 2006.

[57] J. Xiang, A. Vidan, M. Tinkham, R. M. Westervelt, and C. M. Lieber, "Ge/Si nanowire mesoscopic Josephson junctions," Nature nanotechnology, vol. 1, no. 3, pp. 208-213, 2006.
[58] J. Xiang, W. Lu, Y. Hu, Y. Wu, H. Yan, and C. M. Lieber, "Ge/Si nanowire heterostructures as high-performance field-effect transistors," Nature, vol. 441, no. 7092, pp. 489-493, 2006.

[59] O. Hayden, R. Agarwal, and C. M. Lieber, "Nanoscale avalanche photodiodes for highly sensitive and spatially resolved photon detection," Nature Materials, vol. 5, no. 5, pp. 352-356, 2006.

[60] Z. Zhong, Y. Fang, W. Lu, and C. M. Lieber, "Coherent single charge transport in molecular-scale silicon nanowires," Nano Letters, vol. 5, no. 6, pp. 1143-1146, 2005.

[61] Z. H. Zhong, Y. Fang, C. Yang, W. Lu, and C. M. Lieber, "PHYS 1-single charge transport studies in silicon nanowires," in Proceedings of the 229th ACS National Meeting, San Diego, Calif, USA, March 2005.

[62] C. Yang, Z. Zhong, and C. M. Lieber, "Materials science: encoding electronic properties by synthesis of axial modulation-doped silicon nanowires," Science, vol. 310, no. 5752, pp. 1304-1307, 2005.

[63] S. Gradečak, F. Qian, Y. Li, H. G. Park, and C. M. Lieber, "GaN nanowire lasers with low lasing thresholds," Applied Physics Letters, vol. 87, no. 17, Article ID 173111, 3 pages, 2005.

[64] F. Qian, S. Gradečak, Y. Li, C. Y. Wen, and C. M. Lieber, "Core/multishell nanowire heterostructures as multicolor, high-efficiency light-emitting diodes," Nano Letters, vol. 5, no. 11, pp. 2287-2291, 2005.

[65] Y. Cui, X. Duan, J. Hu, and C. M. Lieber, "Doping and electrical transport in silicon nanowires," Journal of Physical Chemistry B, vol. 104, no. 22, pp. 5215-5216, 2000.

[66] Y. Cui and C. M. Lieber, "Functional nanoscale electronic devices assembled using silicon nanowire building blocks," Science, vol. 291, no. 5505, pp. 851-853, 2001.

[67] Y. Cui, Q. Wei, H. Park, and C. M. Lieber, "Nanowire nanosensors for highly sensitive and selective detection of biological and chemical species," Science, vol. 293, no. 5533, pp. 1289-1292, 2001.

[68] J. Q. Hu, X. L. Ma, N. G. Shang et al., "Large-scale rapid oxidation synthesis of $\mathrm{SnO} 2$ nanoribbons," Journal of Physical Chemistry B, vol. 106, no. 15, pp. 3823-3826, 2002.

[69] J. Hu, T. W. Odom, and C. M. Lieber, "Chemistry and physics in one dimension: synthesis and properties of nanowires and nanotubes," Accounts of Chemical Research, vol. 32, no. 5, pp. 435-445, 1999.

[70] N. M. Hwang, W. S. Cheong, D. Y. Yoon, and D. Y. Kim, "Growth of silicon nanowires by chemical vapor deposition: approach by charged cluster model," Journal of Crystal Growth, vol. 218, no. 1, pp. 33-39, 2000.

[71] A. M. Morales and C. M. Lieber, "A laser ablation method for the synthesis of crystalline semiconductor nanowires," Science, vol. 279, no. 5348, pp. 208-211, 1998.

[72] D. Wang and H. Dai, "Low-temperature synthesis of singlecrystal germanium nanowires by chemical vapor deposition," Angewandte Chemie, vol. 40, no. 24, pp. 4783-4786, 2002.

[73] Y. F. Zhang, Y. H. Tang, C. Lam et al., "Bulk-quantity Si nanowires synthesized by $\mathrm{SiO}$ sublimation," Journal of Crystal Growth, vol. 212, no. 1-2, pp. 115-118, 2000.

[74] Y. F. Zhang, Y. H. Tang, N. Wang, C. S. Lee, I. Bello, and S. T. Lee, "Germanium nanowires sheathed with an oxide layer," Physical Review B, vol. 61, no. 7, pp. 4518-4521, 2000.

[75] Y. F. Zhang, Y. H. Tang, N. Wang et al., "Silicon nanowires prepared by laser ablation at high temperature," Applied Physics Letters, vol. 72, no. 15, pp. 1835-1837, 1998.

[76] X. Duan and C. M. Lieber, "General synthesis of compound semiconductor nanowires," Advanced Materials, vol. 12, no. 4, pp. 298-302, 2000. 
[77] X. Duan and C. M. Lieber, "Laser-assisted catalytic growth of single crystal GaN nanowires," Journal of the American Chemical Society, vol. 122, no. 1, pp. 188-189, 2000.

[78] X. Duan, J. Wang, and C. M. Lieber, "Synthesis and optical properties of gallium arsenide nanowires," Applied Physics Letters, vol. 76, no. 9, pp. 1116-1118, 2000.

[79] W. S. Shi, Y. F. Zheng, N. Wang, C. S. Lee, and S. T. Lee, "Oxide-assisted growth and optical characterization of gallium-arsenide nanowires," Applied Physics Letters, vol. 78, no. 21, pp. 3304-3306, 2001.

[80] R. Jalilian, G. U. Sumanasekera, H. Chandrasekharan, and M. K. Sunkara, "Phonon confinement and laser heating effects in Germanium nanowires," Physical Review B, vol. 74, no. 15, Article ID 155421, 6 pages, 2006.

[81] Y. Wang, R. Zhang, T. Frauenheim, and T. A. Niehaus, "Atomistic simulations of self-trapped exciton formation in silicon nanostructures: the transition from quantum dots to nanowires," Journal of Physical Chemistry C, vol. 113, no. 30, pp. 12935-12938, 2009.

[82] K. Nishio, T. Ozaki, T. Morishita, W. Shinoda, and M. Mikami, "Electronic and optical properties of polyicosahedral Si nanostructures: a first-principles study," Physical Review B, vol. 77, no. 7, Article ID 075431, 13 pages, 2008.

[83] C. Harris and E. P. O’Reilly, "Nature of the band gap of silicon and germanium nanowires," Physica E, vol. 32, no. 1-2, pp. 341-345, 2006.

[84] I. Ponomareva, M. Menon, D. Srivastava, and A. N. Andriotis, "Structure, stability, and quantum conductivity of small diameter silicon nanowires," Physical Review Letters, vol. 95, no. 26, Article ID 265502, 4 pages, 2005.

[85] H. Sakaki, "Scattering suppression and high-mobility effect of size-quantized electrons in ultrafine semiconductor wire structures," Japanese Journal of Applied Physics, vol. 19, no. 12, pp. L735-L738, 1980.

[86] S. M. Koo, A. Fujiwara, J. P. Han, E. M. Vogel, C. A. Richter, and J. E. Bonevich, "High inversion current in silicon nanowire field effect transistors," Nano Letters, vol. 4, no. 11, pp. 2197-2201, 2004.

[87] Y. Cui, Z. Zhong, D. Wang, W. U. Wang, and C. M. Lieber, "High performance silicon nanowire field effect transistors," Nano Letters, vol. 3, no. 2, pp. 149-152, 2003.

[88] X. Zhao, C. M. Wei, L. Yang, and M. Y. Chou, "Quantum confinement and electronic properties of silicon nanowires," Physical Review Letters, vol. 92, no. 23, Article ID 236805, 4 pages, 2004.

[89] S. Cahangirov and S. Ciraci, "First-principles study of GaAs nanowires," Physical Review B, vol. 79, no. 16, Article ID 165118, 8 pages, 2009.

[90] D. Csontos, P. Brusheim, U. Zülicke, and H. Q. Xu, "Spin$3 / 2$ physics of semiconductor hole nanowires: valence-band mixing and tunable interplay between bulk-material and orbital bound-state spin splittings," Physical Review B, vol. 79, no. 15, Article ID 155323, 16 pages, 2009.

[91] Y. H. Zhu and J. B. Xia, "Electronic structure of Mn-doped ZnO quantum wires: a mean-field theory study," Physical Review B, vol. 75, no. 20, Article ID 205113, 5 pages, 2007.

[92] Y. Zheng, C. Rivas, R. Lake, K. Alam, T. B. Boykin, and G. Klimeck, "Electronic properties of silicon nanowires," IEEE Transactions on Electron Devices, vol. 52, no. 6, pp. 10971103, 2005.

[93] H. Scheel, S. Reich, and C. Thomsen, "Electronic band structure of high-index silicon nanowires," Physica Status Solidi B, vol. 242, no. 12, pp. 2474-2479, 2005.
[94] M. Kazan, G. Guisbiers, S. Pereira et al., "Thermal conductivity of silicon bulk and nanowires: effects of isotopic composition, phonon confinement, and surface roughness," Journal of Applied Physics, vol. 107, no. 8, Article ID 083503, 14 pages, 2010.

[95] M. Hu, K. P. Giapis, J. V. Goicochea, X. Zhang, and D. Poulikakos, "Significant reduction of thermal conductivity in Si/Ge core-shell nanowires," Nano Letters, vol. 11, no. 2, pp. 618-623, 2011.

[96] D. Donadio and G. Galli, "Temperature dependence of the thermal conductivity of thin silicon nanowires," Nano Letters, vol. 10, no. 3, pp. 847-851, 2010.

[97] L. Liu and X. Chen, "Effect of surface roughness on thermal conductivity of silicon nanowires," Journal of Applied Physics, vol. 107, no. 3, Article ID 033501, 5 pages, 2010.

[98] P. Martin, Z. Aksamija, E. Pop, and U. Ravaioli, "Impact of phonon-surface roughness scattering on thermal conductivity of thin Si nanowires," Physical Review Letters, vol. 102, no. 12, Article ID 125503, 4 pages, 2009.

[99] X. Lü, "Lattice thermal conductivity of Si nanowires: effect of modified phonon density of states," Journal of Applied Physics, vol. 104, no. 5, Article ID 054314, 2008.

[100] A. L. Moore, S. K. Saha, R. S. Prasher, and L. Shi, "Phonon backscattering and thermal conductivity suppression in sawtooth nanowires," Applied Physics Letters, vol. 93, no. 8, Article ID 083112, 3 pages, 2008.

[101] I. Ponomareva, D. Srivastava, and M. Menon, "Thermal conductivity in thin silicon nanowires: phonon confinement effect," Nano Letters, vol. 7, no. 5, pp. 1155-1159, 2007.

[102] T. Thonhauser and G. D. Mahan, "Phonon modes in Si [111] nanowires," Physical Review B, vol. 69, no. 7, Article ID 075213, 5 pages, 2004.

[103] S. Bhattacharyya and S. Samui, "Phonon confinement in oxide-coated silicon nanowires," Applied Physics Letters, vol. 84, no. 9, pp. 1564-1566, 2004.

[104] S. Piscanec, M. Cantoro, A. C. Ferrari et al., "Raman spectroscopy of silicon nanowires," Physical Review B, vol. 68, no. 24, Article ID 241312, 4 pages, 2003.

[105] R. K. Ray et al., "Temperature dependence of Raman scattering in germanium," in Proceedings of the 2nd International Conference on Light Scattering in solids, R. Balkanski, Ed., Flammarion, Paris, France, 1971.

[106] H. Richter, Z. P. Wang, and L. Ley, "The one phonon Raman spectrum in microcrystalline silicon," Solid State Communications, vol. 39, no. 5, pp. 625-629, 1981.

[107] I. H. Campbell and P. M. Fauchet, "The effects of microcrystal size and shape on the one phonon Raman spectra of crystalline semiconductors," Solid State Communications, vol. 58, no. 10, pp. 739-741, 1986.

[108] P. Alfaro, R. Cisneros, M. Bizarro, M. Cruz-Irisson, and C. Wang, "Raman scattering by confined optical phonons in $\mathrm{Si}$ and Ge nanostructures," Nanoscale, vol. 3, no. 3, pp. 1246$1251,2011$.

[109] N. Fukata, T. Oshima, N. Okada et al., "Phonon confinement in silicon nanowires synthesized by laser ablation," Physica B, vol. 376-377, no. 1, pp. 864-867, 2006.

[110] A. K. Arora, M. Rajalakshmi, T. R. Ravindran, and V. Sivasubramanian, "Raman spectroscopy of optical phonon confinement in nanostructured materials," Journal of Raman Spectroscopy, vol. 38, no. 6, pp. 604-617, 2007.

[111] S. Sahoo, S. Dhara, S. Mahadevan, and A. K. Arora, "Phonon confinement in stressed silicon nanocluster," Journal of $\mathrm{Na}$ noscience and Nanotechnology, vol. 9, no. 9, pp. 5604-5607, 2009. 
[112] W. S. O. Rodden, C. M. S. Torres, and C. N. Ironside, "Threedimensional phonon confinement in CdSe microcrystallites in glass," Semiconductor Science and Technology, vol. 10, no. 6, pp. 807-812, 1995.

[113] S. Khachadorian, H. Scheel, A. Colli, A. Vierck, and C. Thomsen, "Temperature dependence of first- and second-order Raman scattering in silicon nanowires," Physica Status Solidi B, vol. 247, no. 11-12, pp. 3084-3088, 2010.

[114] P. R. Stoddart and J. D. Comins, "Quasielastic light scattering in silicon," Physical Review B, vol. 62, no. 23, pp. 1538315385,2000

[115] D. Li, Y. Wu, P. Kim, L. Shi, P. Yang, and A. Majumdar, "Thermal conductivity of individual silicon nanowires," Applied Physics Letters, vol. 83, no. 14, pp. 2934-2936, 2003.

[116] A. I. Hochbaum, R. Chen, R. D. Delgado et al., "Enhanced thermoelectric performance of rough silicon nanowires," Nature, vol. 451, no. 7175, pp. 163-167, 2008.

[117] P. A. Temple and C. E. Hathaway, "Multiphonon Raman spectrum of silicon," Physical Review B, vol. 7, no. 8, pp. 3685-3697, 1973.

[118] H. Bilz, Phonon Dispersion Relations in Insulators, Springer, Berlin, Germany, 1979.

[119] M. Rajalakshmi, A. K. Arora, B. S. Bendre, and S. Mahamuni, "Optical phonon confinement in zinc oxide nanoparticles," Journal of Applied Physics, vol. 87, no. 5, pp. 2445-2448, 2000.

[120] D. D. D. Ma, C. S. Lee, F. C. K. Au, S. Y. Tong, and S. T. Lee, "Small-diameter silicon nanowire surfaces," Science, vol. 299, no. 5614, pp. 1874-1877, 2003.

[121] S. Khachadorian, K. Papagelis, H. Scheel, A. Colli, A. C. Ferrari, and C. Thomsen, "High pressure Raman scattering of silicon nanowires," Nanotechnology, vol. 22, no. 19, Article ID 195707, 2011.

[122] S. Khachadorian, H. Scheel, M. Cantoro, A. Colli, A. C. Ferrari, and C. Thomsen, "The morphology of silicon nanowire samples: a Raman study," Physica Status Solidi B, vol. 246, no. 11-12, pp. 2809-2812, 2009.

[123] H. Scheel, S. Khachadorian, M. Cantoro, A. Colli, A. C. Ferrari, and C. Thomsen, "Silicon nanowire optical Raman line shapes at cryogenic and elevated temperatures," Physica Status Solidi B, vol. 245, no. 10, pp. 2090-2093, 2008.

[124] J. Wu, D. Zhang, Q. Lu, H. R. Gutierrez, and P. C. Eklund, "Polarized Raman scattering from single GaP nanowires," Physical Review B, vol. 81, no. 16, Article ID 165415, 8 pages, 2010.

[125] H. Scheel, S. Reich, A. C. Ferrari, M. Cantoro, A. Colli, and C. Thomsen, "Raman scattering on silicon nanowires: the thermal conductivity of the environment determines the optical phonon frequency," Applied Physics Letters, vol. 88, no. 23, Article ID 233114, 3 pages, 2006.

[126] R. Gupta, Q. Xiong, C. K. Adu, U. J. Kim, and P. C. Eklund, "Laser-induced fano resonance scattering in silicon nanowires," Nano Letters, vol. 3, no. 5, pp. 627-631, 2003.

[127] T. Kanata, H. Murai, and K. Kubota, "Raman and x-ray scattering from ultrafine semiconductor particles," Journal of Applied Physics, vol. 61, no. 3, pp. 969-971, 1987.

[128] M. Balkanski, R. F. Wallis, and E. Haro, "Anharmonic effects in light scattering due to optical phonons in silicon," Physical Review B, vol. 28, no. 4, pp. 1928-1934, 1983.

[129] H. Tang and I. P. Herman, "Raman microprobe scattering of solid silicon and germanium at the melting temperature," Physical Review B, vol. 43, no. 3, pp. 2299-2304, 1991.

[130] H. H. Burke and I. P. Herman, "Temperature dependence of Raman scattering in Ge1-xSix alloys," Physical Review B, vol. 48, no. 20, pp. 15016-15024, 1993.
[131] B. Li, D. Yu, and S. L. Zhang, "Raman spectral study of silicon nanowires," Physical Review B, vol. 59, no. 3, pp. 1645-1648, 1999.

[132] P. Mishra and K. P. Jain, "First- and second-order Raman scattering in nanocrystalline silicon," Physical Review B, vol. 64, no. 7, Article ID 073304, 4 pages, 2001.

[133] A. L. Arora, M. Rajalakshmi, and T. R. Ravindran, "Phonon confinement in nanomaterials," in Encyclopedia of Nanoscience and Technology, vol. 8, pp. 499-512, American Scientific Publisher, 2004.

[134] C. M. S. Torres, A. Zwick, F. Poinsotte et al., "Observations of confined acoustic phonons in silicon membranes," Physica Status Solidi C, vol. 1, no. 11, pp. 2609-2612, 2004.

[135] X. Lü, J. H. Chu, and W. Z. Shen, "Modification of the lattice thermal conductivity in semiconductor rectangular nanowires," Journal of Applied Physics, vol. 93, no. 2, pp. 1219-1229, 2003.

[136] X. Lü and J. Chu, "Lattice thermal conductivity in a silicon nanowire with square cross section," Journal of Applied Physics, vol. 100, no. 1, Article ID 014305, 6 pages, 2006.

[137] Y. Chen, D. Li, J. R. Lukes, and A. Majumdar, "Monte Carlo simulation of silicon nanowire thermal conductivity," Journal of Heat Transfer, vol. 127, no. 10, pp. 1129-1137, 2005. 

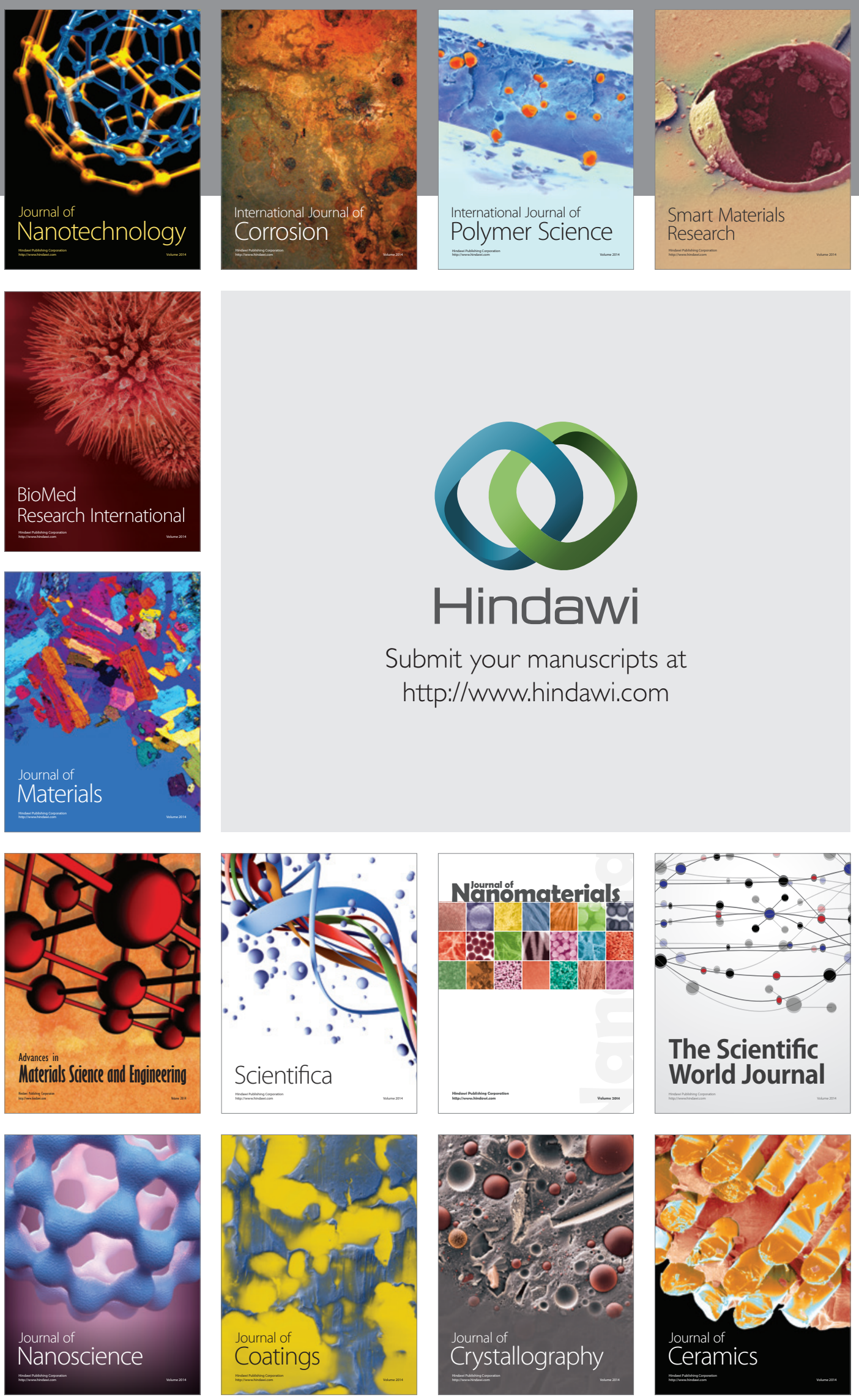

The Scientific World Journal

Submit your manuscripts at

http://www.hindawi.com

\section{World Journal}

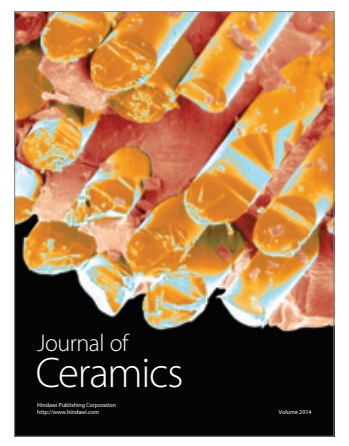


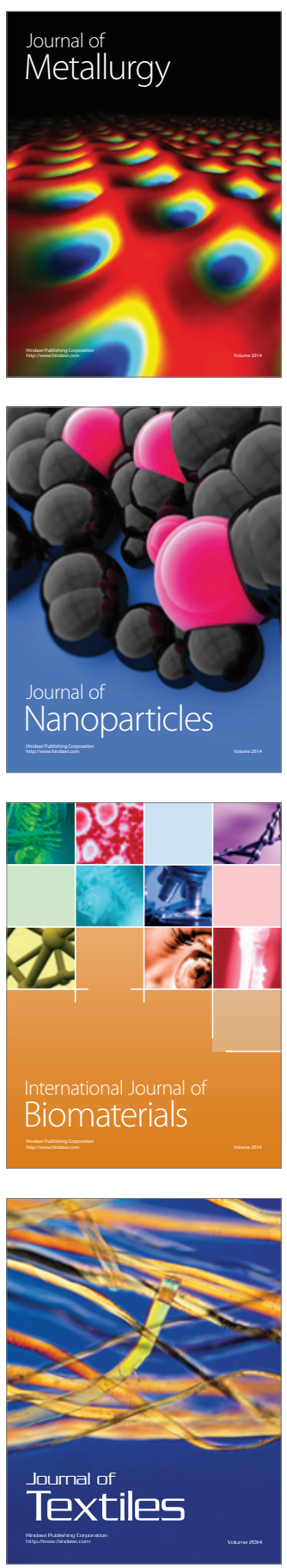